\title{
Learning from Others? Decision Rights, Strategic Communication, and Reputational Concerns*
}

\author{
Otto H. Swank and Bauke Visser \\ Erasmus School of Economics and Tinbergen Institute
}

May 2010

\begin{abstract}
Decision-makers can learn from their own past experience, and, potentially, from the experience of other decision-makers who face similar problems. When experience is private information, communication is necessary to learn from others. In a twoperiod model in which decision-makers care about their reputation, we study how the assignment of decision rights (who decides on the actions taken in period two?) and the information on which reputations are based determine (i) the willingness to share information, (ii) decisions, and (iii) welfare. Centralizing decision rights may hurt welfare due to the negative effect on the quality of communication. The welfare effects of reputation depend on whether perceptions of a decision-maker's ability are based solely on his own action or on comparisons across decision-makers, and on the assignment of decision rights.
\end{abstract}

Keywords: centralization, decentralization, authority, learning, cheap talk, reputational concerns, policy diffusion

\footnotetext{
*We thank seminar audiences at Bologna University, the European University Institute, Maastricht University, MEDS Kellogg School of Management, Tilburg University, and at the MPSA 2009 conference, and especially Michael Raith and Vladimir Karamychev for their comments and questions. A previous version of this paper was called Decision Making and Learning in a Globalizing World. Part of this paper was written while Visser was a Fernand Braudel fellow at the EUI. Email: swank@ese.eur.nl and bvisser@ese.eur.nl
} 
"Changing on the basis of new evidence means accepting the uncomfortable notion that we [doctors] did it wrong, or less well, before. Thus we needlessly harmed people in the past. This is painful for health professionals, (...) even if our actions were unintentional or the evidence didn't exist previously. Some find it easy to say 'Well, better stop harming now than carry on,' but denial is simpler, powerful, and comforting"

\section{Introduction}

Learning from one's own experience and learning from others are two important ways in which decision-makers can improve the decisions they take over time. It may help a physician in identifying a better intervention for a patient with a given diagnosis; it may help police in establishing a more effective way of criminal investigative analysis; it can help organizational divisions in establishing what customer-relationship management system works best, etc. The challenge in each case is to recognize the best course of action and to ensure its diffusion.

In practice, the identification and diffusion of the best course of action raise two main problems. First, it has been established that once a decision-maker has chosen a course of action, he tends to cling to it, even if subsequently his own experience shows that another action would likely result in a better outcome. ${ }^{2}$ One important reason for this behaviour has been put forward by, e.g., Kanodia et al. (1989), and Prendergast and Stole (1996): the presence of reputational concerns. Changing course of action amounts to an admission that the previous action was inappropriate. As a result, a change affects perceptions of the ability of the decision-maker adversely. A decision-maker who wants to acquire a reputation for identifying the correct action, will be hesitant to change. The second problem is that learning from decision-makers located at other sites (hospitals, states, divisions etc.) is not automatic, but requires their willingness to share their private information. Reputational concerns may make communication strategic.

In this paper, we study how reputational concerns influence the quality of learning from others. We present a simple two-period model of learning. In period one, each agent at

\footnotetext{
${ }^{1}$ Susan Bewley, consultant obstetrician, in Getting to the bottom of evidence based medicine, the British Medical Journal, April 5, 2008.

${ }^{2}$ See, e.g., Thaler (1980).
} 
his own site is confronted with a common problem, and receives a private signal. The informativeness of the signal is determined by the agent's ability at identifying the better course of action. Unaware that others are struggling with the same problem, each agent optimally follows his private signal, and next privately learns the true, common value of the chosen course of action. It may be that agents receive the same signal. The outcome of period one is a "historical pattern" of actions taken to address the common problem.

Next, decisions have to be made as to the action to adopt in period two. An agent may rely only on his own experience - the case studied in Prendergast and Stole. But if there is an awareness that other agents have addressed the same problem, it might be benificial to make use of their experience. This requires communication about locally gained experience. Inspired by real world examples that we discuss below, our analysis focuses on two dimensions that may influence the quality of learning.

(i) Second period decision rights. Do local agents keep the authority to decide in period two (decentralized learning), or is it in the hands of some "center" that decides what actions are taken at the different sites (centralized learning)? In the first case, communication is horizontal, among the local agents. In the latter case, communication is vertical, from local agents to the center.

(ii) Information on which the perception of a local agent's ability is based. As in Prendergast and Stole, we assume that perceptions are based on observed actions only, not the values these actions generate, but we distinguish two cases. The perception of an agent's ability is either based on the actions taken at his site (locally determined reputations), or on the actions taken at all sites (globally determined reputations). In the latter case, comparisons across sites become possible, thanks to, e.g., increased transparency. As highly able agents are more likely to initially take the same action than less able ones, such comparisons may affect perceptions.

We assume that the utility of a local agent is increasing in the value of the action taken at his site and his end-of-period reputation, and that the centre (e.g. a health care body, the head of the police force, corporate headquarters) only cares about the value of the actions taken. We compare various learning environments (as characterized by the two dimensions just mentioned) in terms of the ex ante expected value of the actions taken in period two 
('welfare'). As there may be conflicts of interest between local agents, and between local agents and the center communication about the experiences gained is strategic. The only formal mechanism in place are the decision rights in period two. In particular, a decisionmaker cannot commit to make use of the information he will receive in a specific way. As a result, communication about the privately gained experience amounts to cheap talk.

The goal of this paper is to further our understanding of learning processes by establishing how (i) the assignment of decision rights and (ii) the information on which perceptions of abilities are based jointly determine the willingness of decision-makers to share private information, the quality of the decisions taken conditional on the information transmitted, and overall welfare.

As said, in practice the identification and diffusion of the best course of action can be a struggle. Consider the medical profession. The delivery of medical interventions varies widely from place to place. ${ }^{3}$ This variation has been a source of worries as, most likely, some patients do not receive optimal treatment. ${ }^{4}$ It also offers scope for learning. In response, physicians' associations and health care authorities have exerted much effort to design learning processes in which locally gained experiences are compared, and best practices - interventions, surgical procedures, drug use - diffused. In the medical sector, expert panels are frequently used to evaluate the evidence on the effectiveness of rival practices in a given field. Given the close ties between experts and industry, and the long gestation period that characterizes the development of practices, experts tend to have vested interests and to identify with certain practices. The result, according to students of expert panels, is "process loss" due to reputational concerns, leading in turn to poor information exchange and aggregation in the meetings, and a low adoption rate of best practices afterwards. ${ }^{5}$ Organizing these panels is therefore fraught with problems. An important organizational dimension is the degree of centralization of the process and, relatedly, the degree of freedom individual physicians have in following the outcomes of panel meetings. ${ }^{6}$ It also seems that the IT revolution and increased information dissemination over the internet, in combination

\footnotetext{
${ }^{3}$ That variation is large is a well-established fact, see Phelps (2000).

${ }^{4}$ See, e.g., Eddy (1990).

${ }^{5}$ See Fink et al. (1984) and Rowe et al. (1991).

${ }^{6}$ Eddy (1990) distinguishes, in increasing degree of freedom, standards, guidelines, and options.
} 
with societal pressure to increase transparency makes it easier for patients and authorities to compare medical practices across places. This information can then shape the perception of physicians' abilities.

The European Union is another case in point. It has been promoting the so-called open method of coordination (OMC) to foster learning and the diffusion of best practices in many policy areas. The hope is that goals like EU competitiveness can be furthered by avoiding the grand questions about the best model for Europe and by taking instead a more pragmatic micro-orientation in which countries that face similar problems seek to learn from each other. ${ }^{7}$ Rather than relying on legislation by Brussels-a form of centralized decision-making--, the OMC leaves decision rights with the EU countries: they decide whether to implement the lessons learned. Moreover, instead of applying formal sanctions to transgressors, the OMC turns to naming and shaming to expose a country's weak performance in public, and applies peer pressure if a country opposes adoption of superior policies. ${ }^{8}$ In practice, the method is not considered to be very successful in guaranteeing a high quality learning process. It is generally felt that countries exaggerate the success of their current practices. Also, the implementation of new ideas is very limited. Claudio Radaelli (2003, p. 12) argues that these disappointing results stem from a misguided view of policy makers among the proponents of the OMC. Rather than caring about the truth, they care about 'political capital' and 'prestige' - forms of reputational concerns. Arguably, the 'naming and shaming in public' suggests that the perception of an agent's ability in the case of the OMC can be based on comparisons across countries.

We obtain the following results. Our model replicates one of the finding of Prendergast and Stole: if an agent cannot communicate with another agent but has to decide what course of action to take in complete isolation and on the basis of his own experience only, then reputational concerns make him conservative. ${ }^{9}$ This conservatism implies a loss of welfare that is growing in the degree to which the agent cares about his perceived ability.

\footnotetext{
${ }^{7}$ Policy areas where the OMC has been applied in areas as diverse as employment, social inclusion, innovation, education, occupational health and safety.

${ }^{8}$ See Pochet (2005) and Radaelli (2003).

${ }^{9}$ As ours is a binary-choice model, we cannot replicate the second main finding of Prendergast and Stole, namely that early on a decision-maker reacts too strongly to information to boost his reputation.
} 
If learning from others is possible and happens in a decentralized way, an agent remains conservative to protect his reputation. The information on which he can base his second period decision is partly gained from own experience and from what others are willing to share with him. The quality of information exchange is high if reputations are locally determined. He can only gain by listening to others, and has nothing to loose by truthfully revealing his own experience, as his reputation does not depend on the action that others adopt in period two. If instead reputations are determined globally, his reputation is particularly strong if others start to adopt "his" initial course of action. As a result, communication becomes strategic: it becomes important for an agent to convince others that "his" technology is best. We show that communication breaks down completely. Interestingly, the fact that an agent has less information on which to base his second period decision does not by itself mean that welfare goes down. This is also determined by the reputational gain of distorting the decision. Essentially, if there is more information on which the agent bases his decision, there is also more information about his ability that can be gleaned from that decision. We derive an intuitive condition that specifies when the additional information shared among agents next leads to an increase or decrease in welfare.

Second period decision-making in case of a centralized process does not suffer from conservatism as, by assumption, the center only cares about welfare. But the center depends on the agents to provide him with information. An agent now faces a trade-off. On the one hand, as the agent has no decision-making power, he wants to make sure that the center is well-informed. On the other hand, his reputational concerns imply that he wants the center to impose "his" technology at either site. In equilibrium, each agent sends coarse information about his own practice. This has a number of consequences. First, replacing a decentralized process by a centralized one reduces the quality of information exchange if reputations are locally determined. We derive the conditions under which the quality of information exchange in case of a centralized process becomes so poor that it offsets the improved decision-making conditional on information. Second, if reputations are globally determined, replacing a decentralized process by a centralized one improves communication. In fact, a centralized process creates more welfare than a decentralized one in case reputations are based on comparisons across sites. We show that communication between the 
agents and the center never vanishes, for any finite weight the agents put on their reputations. Finally, we derive conditions under which, in case of centralized process, welfare goes up if locally based reputations give way to globally based reputations.

The paper is organized as follows. The next section discusses the related literature. In Section 3, we present the model. Section 4 analyses isolated agents, a benchmark situation in which agents can learn from their own past experience only. In section 5 we analyse decentralized learning, with local and global markets. In section 6 we perform the same analysis for centralized learning. Section 7 contains the comparisons. Section 8 concludes.

\section{Related Literature}

Our paper is related to a number of literatures.

(1) Information processing when information is dispersed. Our paper is closest related to Alonso, Dessein, and Matouschek (2008) and to Rantakari (2008). They study the desirability of a centralized or decentralized process in the context of a multidivisional firm. Each division benefits from adapting its decision to its own market circumstances and from coordinating its decision with those of the other divisions. Divisions are privately informed about their market circumstances. They can either exchange information and next decide independently of each other what decisions to take or they can report information to headquarters which then decides for both divisions. They show that even if coordination becomes of overriding concern to the firm, a decentralized process may still outperform a centralized process due to the difference in quality of communication. ${ }^{10}$ As Alonso et al. and Rantakari we study the effect of the assignment of decision rights on the quality of communication and of the final decisions taken. The situation we analyse, however, is quite different. In our paper, there are no local circumstances to which a decision should ideally be adapted, nor is there a need to coordinate per se. Instead, there is room for learning from each other's past experience (to identify the better course of action), resistance to change

\footnotetext{
${ }^{10}$ Friebel and Raith (2010) study how the scope of the firm affects the quality of strategic information transmission between a division and head quarters.
} 
(because of reputational concerns), and possibly the desire to convince other agents to adopt one's initial course of action (again, due to reputational concerns).

The importance of dispersed information has already been highlighted in debates on the relative merits of a planning economy and a market economy in the 1930s, see e.g. Hayek (1945). Team theory, as developed by Marshak and Radner (1972), is one of the first formal attempts to address the question how an organization should be structured to deal optimally with dispersed information. In this theory, interests of organizational members are perfectly aligned, and so incentive problems do not arise. Instead, the focus is on exogenously specified communication and information-processing constraints. In our paper, we focus on the effect of agents' interests on their willingness to share information. We share with the mechanism design literature a focus on the incentive problems surrounding communication. However, we do not assume that agents can commit to mechanisms. Only decision rights can be assigned. As a result, an important implication of the Revelation Principle, that a centralized process is always at least as good as a decentralized one, does not hold. ${ }^{11}$

There are other papers in economics and political science that explore how characteristics of decision-making processes influence the quality of cheap talk communication. ${ }^{12}$. The current paper differs from the existing literature in its focus on the possibilities for learning from one's own experience and from the experience of others in a context where agents have reputational concerns.

(2) Reputational concerns. The effect of reputational or career concerns has been studied in various environments. Holmstrom (1999) studies the incentives such concerns give to exert productive effort if there is uncertainty about an agent's ability level. If there is uncertainty about an agent's ability to 'read' or predict the state of the world one speaks of 'expert' models. Experts use the recommendations that they give, the implementation decision that

\footnotetext{
${ }^{11}$ See Mookherjee (2006) and Poitevin (2000) for excellent surveys of the assumptions underlying the Revelation Principle. They also discuss various modelling strategies that can be used to explain why decentralization and delegation outperform centralization.

${ }^{12}$ In economicss see e.g. Dessein (2002, 2007), Visser and Swank (2007), Alonso et al. (2008), Rantakari (2008), and Friebel and Raith (2010). In political science, see e.g., Gilligan and Krehbiel (1987), AustenSmith (1990), Coughlan (2000), and Austen-Smith and Feddersen (2005).
} 
they take, or the effort they exert to convince the market of their expertise. ${ }^{13}$ Part of this expert literature looks at the effects of information disclosure ('transparency') about an expert's actions and about the outcomes of decisions. ${ }^{14}$ The present paper is related to that literature, as the information on which an agent's reputation is based can change, either by design or by some external force, from specific to his site to involving comparisons across sites. We show that as a result of the additional information, communication is destroyed in case of a decentralized process, but improves in case of a centralized process. That is, the same form of transparency may give rise to very different effects depending on the institutions in which it is introduced.

(3) Laboratory federalism and policy diffusion. ${ }^{15}$ In an interesting recent paper, Volden, Ting, and Carpenter (2008) study what happens if policy makers trade off policy effectiveness at solving problems and political preferences. They compare the adoption patterns of states that act independently and learn from their own past performance at addressing common problems with the patterns that arise if states learn from each other. Our focus is different from theirs as we study the quality of information exchange among decisionmakers, compare centralized and decentralized decision-making, and study the effect of the informational basis of reputations.

(4) Learning. We already mentioned the seminal paper by Prendergast and Stole (2006) on learning from one's own observations by an agent who also cares about his perceived ability. Compared to their paper, we introduce learning from others, and hence communication, a discussion of decision rights, and different information sets on which perceptions of ability can be based. Our paper is also related to some existing literature on learning from others. This literature is, however, methodologically quite different from ours. In the existing literature, it is assumed that either an agent observes the true value of the actions taken by others,

\footnotetext{
${ }^{13}$ Scharfstein and Stein (1990) and Ottaviani and Sorensen (2001, 2006) deal with the advice given by experts. Milbourn et al (2001) and Suurmond et al. (2004), deal with the projects an expert implements and the effort he exerts to become informed.

${ }^{14}$ See Suurmond et al. (2004) and Prat (2005) in a single-agent setting, and Levy (2007) and Swank and Visser (2009) in a committee setting.

${ }^{15}$ See Oates (1999) for a survey.
} 
whether the environment is strategic ${ }^{16}$ or not ${ }^{17}$, or that no such information is observed at all ${ }^{18}$. Furthermore, inertia or conservatism is an exogenous factor. For example, in the literature on word-of-mouth communication, it is assumed that only a given fraction of agents updates its decisions once new information becomes available. In our paper both the quality of the information exchange and the degree of inertia are equilibrium outcomes. Were it not for the reputational concerns, the problem the agents are facing in our model, that of choosing one technology out of many, is similar to a common value bandit problem in which the bandit's arms represent the technologies of unknown, but common, value. ${ }^{19}$ The main difference is that in a bandit problem the distribution of the value of a technology does not change with an observation of the value of another technology, whereas in our problem it does. This stems from the fact that in our model the initial signal an agent receives provides information about the better technology. The higher is the observed value of a technology $Y$, the higher is the probability that the agent identified the better technology. And this means that it becomes more likely that the value of the other technology is lower than the actual value of $Y$.

The fact that in our model the quality of information exchange and the degree of inertia are endogenous, and that a key assumption of the statistical bandit model is violated imply that a general analysis of the asymptotic behaviour of the decision-making processes described here is difficult and beyond the scope of this paper. Instead, we compare the

\footnotetext{
${ }^{16}$ See the discussion of social learning in a strategic experimentation game in Bergemann and Välimäki (2006). In this literature, it is assumed that an agent perfectly observes both the technology others use and the true value they obtain. It is not clear that an agent, if he could, would not want to deviate from a strategy of truthfully revealing the value of the technology he has gained experience with. It seems that he would benefit from exaggerating the value as this would make adoption by others more likely. As a result, more (public) information would become available about this technology, and the deviator would benefit from an improved estimate of the technology's value.

${ }^{17}$ See Bala and Goyal (1998) for a model of learning in non-strategic networks, and Ellison and Fudenberg (1993, 1995) and Banerjee and Fudenberg (2004) for analyses of word-of-mouth communication in nonstrategic environments.

${ }^{18}$ In the literature on informational herding, communication between decision-makers is excluded although the environment in non-strategic. See e.g. Bikhchandani, Hirshleifer and Welch (1998). See Çelen, Kariv and Schotter (2008) for a first experimental analysis of social learning from actions and advice.

${ }^{19}$ See Bergemann and Välimäki (2006) for a concise survey of bandit problems.
} 
behaviour of agents across various decision-making processes in a two-period setting.

(5) Cheap talk. In their seminal paper, Crawford and Sobel (1982) show that cheap talk between an informed sender and an uninformed receiver (decision-maker) can be informative, and that the quality of information exchange depends on the degree of alignment between the interests of both parties. In Crawford and Sobel, and in the literature on cheap talk in general, the degree of alignment is exogenously given. In our model, by contrast, it is determined in equilibrium. The reason is that senders are concerned with their reputations. These reputations are determined in equilibrium. A consequence is that, as we show below, in case of a centralized process and reputations based on comparisons across sites, cheap talk remains informative for any finite weight that agents put on their reputation.

\section{A model of learning from own experience and learn- ing from others with reputational concerns.}

There are two sites (hospitals, states, etc.), $i \in\{1,2\}$, and one problem. There is an agent $i$ at each site. Often, $j$ will denote "the other site" or "the other agent," $j \neq i$. The problem has to be addressed at each site both in period $t=1$ and in $t=2$. There are two possible technologies (policies, interventions, etc.) $X \in\{Y, Z\}$, one of which has to be used to address the problem at each site in each period. The technology adopted at site $i$ in period $t$ is denoted by $X_{i, t}$. A priori, the value of technology $X$ is unknown, but independent of time and site. It is a random draw from a continuous and strictly increasing distribution function $F_{X}(\cdot)$ and associated density function $f_{X}(\cdot)$, with support $[0,1]$. Note that we use $X$ both to denote a technology and its random value. We assume that the values of technologies $Y$ and $Z$ are iid, $F_{Y}=F_{Z}=F$. We use lower case letters, like $x$, to denote a possible value (realization) of the value of technology $X$, such that $x \in[0,1]$. As strategies will be defined in terms of $X$ (or $x$ ), it will be useful to let $X^{C}$ (or $x^{C}$ ) refer to "the other technology". That is, if $X=Y$, then $X^{C}=Z$, etc.

The agents' ability levels $\theta_{i} \in\{\underline{\theta}, \bar{\theta}\}$ and the state of the world $(y, z) \in[0,1]^{2}$ are exogenously given. The ability levels and the state of the world are all statistically independent, 
with $\pi=\operatorname{Pr}\left(\theta_{i}=\bar{\theta}\right) \in(0,1)$ for $i \in\{1,2\}$.

At the beginning of period $t=1$, agent $i$ at site $i$ receives a private, non-verifiable, signal $s_{i} \in\left\{s^{Y}, s^{Z}\right\}$ about which technology solves the problem best. The informativeness of the signal depends on the agent's ability: $\operatorname{Pr}\left(s^{X} \mid x>x^{C}, \bar{\theta}\right)=1, \operatorname{Pr}\left(s^{X} \mid x^{C}>x, \bar{\theta}\right)=0$, $\operatorname{Pr}\left(s^{X} \mid x>x^{C}, \underline{\theta}\right)=\operatorname{Pr}\left(s^{X} \mid x^{C}>x, \underline{\theta}\right)=1 / 2$, for $X \in\{Y, Z\}$. That is, if $i$ is highly able, $\theta_{i}=\bar{\theta}$, the signal reveals with probability one the better technology: $\operatorname{Pr}\left(x>x^{C} \mid s^{X}, \bar{\theta}\right)=1$ for $X \in\{Y, Z\}$. Hence, conditional on $s^{X}$ and $\theta=\bar{\theta}, X$ is distributed as the maximum of two iid random variables, $F_{X}\left(x \mid s^{X}, \bar{\theta}\right)=F(x)^{2}$. On the other hand, if $i$ is less able, $\theta_{i}=\underline{\theta}$, the signal is uninformative about the relative quality of the technology: $F_{X}\left(x \mid s^{X}, \underline{\theta}\right)=F(x)$. Note that an agent does not get a signal about his ability. Instead, $\pi$ is the common prior. ${ }^{20}$ Still in period $1, i$ next decides which technology $X$ to adopt on the basis of his signal $s_{i}$. At the end of the period he learns the value $x$ of the chosen technology.

At this point, it is worth emphasizing that the focus of our analysis will be on period 2. As mentioned in the introduction, we intend to understand the pros and cons of alternative learning processes in situations where (i) agents have gained experiences with different technologies, treatments, or policies and (ii) there is scope for learning from others. In our model, period 1 can be interpreted as the history in which agents gained information. We model history to stress that past decisions matter for current decisions, for example, through reputational concerns.

We distinguish three learning processes $\mathrm{p}$ that characterize period $t=2$. Such a process consists of a decision-making stage, possibly preceded by a communication stage. In case there is a communication stage, agent $i$ sends a message about the quality of the technology adopted at site $i$ in period $t=1$. The receiver of this message depends on the process p. We assume that agent $i$, if and when he sends a message, knows the technology (not its value) that $j$ has used in $t=1$ when he sends a message. This is often the relevant case, as agents may well be aware that other technologies are used, without knowing their quality. Hence, a communication strategy $\mu_{i}^{\mathrm{p}}(\cdot)$ is a conditional probability distribution. Let $\mu_{i}^{\mathrm{p}}\left(m_{i} \mid s_{i}, x_{i, 1}, X_{j, 1}\right)$ be the likelihood that $i$ sends a cheap talk message $m_{i} \in M$, where

\footnotetext{
${ }^{20}$ What matters for the results is that if $\theta_{i}=\bar{\theta}$, member $i$ has a higher likelihood of correctly assessing the state of the economy than if $\theta_{i}=\underline{\theta}$.
} 
$M=[0,1]$ is a message space, in case his signal equals $s_{i}$, the observed value of $X_{i, 1}$ equals $x_{i, 1}$, and agent $j$ uses technology $X_{j, 1}$. Next, a decision maker determines which technology $X_{i, 2}$ is adopted at site $i$ at time $t=2$. Who this decision maker is depends on the decision process p. Let $I_{i}^{\mathrm{p}} \in \mathcal{I}_{i}^{\mathrm{p}}$ be the information this person has at the beginning of the decision-making stage. It depends on the process p. The decision strategy $d_{i}^{\mathrm{p}}$ determines the relationship between $I_{i}^{\mathrm{p}}$ and the technology adopted at site $i$.

(i) In case of isolated agents ( $p=i a)$, an agent is unaware of other agents addressing the same problem, and therefore do not communicate. Hence, $\mathcal{I}_{i}^{\text {ia }}=\left\{s^{Y}, s^{Z}\right\} \times[0,1]$ : the information $i$ has is his signal and the value of the technology used in $t=1$. Agent $i$ decides on $X_{i, 2}$. Let $d_{i}^{\text {ia }}\left(s_{i}, x_{i, 1}\right) \in\{Y, Z\}$ denote the technology that $i$ uses in $t=2$ as a function of his information.

(ii) In case of decentralized learning $(\mathrm{p}=\mathrm{dl})$, each agent $i$ simultaneously sends a message $m_{i}$ to the other agent concerning the value of the technology he has adopted in $t=1$. So, $\mathcal{I}_{i}^{\mathrm{dl}}=\left\{s^{Y}, s^{Z}\right\} \times[0,1] \times M \times\{Y, Z\} \times M$. That is, in addition to the information in case of $\mathrm{p}=\mathrm{ia}$, and the message he sends to $j, i$ also knows the technology $X_{j, 1} \in\{Y, Z\}$ adopted at the other site, and the message $m_{j} \in M$ about the value of that technology. Agent $i$ next decides on $X_{i, 2}$. Let $d_{i}^{\mathrm{dl}}\left(s_{i}, x_{i, 1}, m_{i}, X_{j, 1}, m_{j}\right) \in\{Y, Z\}$ denote the technology that $i$ adopts in $t=2$ given $I_{i}^{\mathrm{dl}}$.

(iii) In case of centralized learning $(\mathrm{p}=\mathrm{cl})$, each agent $i$ simultaneously sends a message $m_{i}$ concerning the value of the technology he has adopted in $t=1$ to "the center." Hence, $\mathcal{I}_{C}^{\mathrm{cl}}=$ $\{Y, Z\}^{2} \times M^{2}$ represents the center's information set: information about which technology has been adopted at each site, and a message concerning the value of each technology. Next, the center decides which technology is adopted at either site. Let $d_{C}^{\mathrm{cl}}\left(X_{1,1}, X_{2,1}, m_{1}, m_{2}\right) \in$ $\{Y, Z\} \times\{Y, Z\}$ denote the correspondence indicating for given technologies used at either site and for given messages sent by the agents the technology that is used at sites 1 and 2, respectively in $t=2$. As no confusion can arise, we write $\mathcal{I}_{C}$ instead of $\mathcal{I}_{C}^{\mathrm{cl}}$, and $d_{C}$ instead of $d_{C}^{\mathrm{cl}}$.

An agent's utility depends on the value of the technology adopted at his site and on his perceived ability or reputation. This perception is based on the information set $\Omega_{i, t}$. We will say that "the market" infers an agent's reputation from $\Omega_{i, t}$. This market could 
be, e.g., the (internal) labour market or the electoral market. As in Prendergast and Stole (1996), we assume that perceptions are based on actions (technologies) chosen, not on the value generated. We distinguish two cases. Say that reputations are locally determined if the reputation of agent $i$ is based on the technologies used at site $i$ only, $\Omega_{i, 1}=\left\{X_{i, 1}\right\}$ and $\Omega_{i, 2}=\left\{X_{i, 1}, X_{i, 2}\right\}$ for $i \in\{1,2\}$. Instead, say that reputations are globally determined if the reputation of agent $i$ is based on the technologies used at both sites $i$ and $j, \Omega_{i, 1}=\left\{X_{i, 1}, X_{j, 1}\right\}$ and $\Omega_{i, 2}=\left\{X_{i, 1}, X_{j, 1}, X_{i, 2}, X_{j, 2}\right\}$ for $i \in\{1,2\}$. We call $\left(X_{i, 1}, X_{j, 1}, X_{i, 2}, X_{j, 2}\right)$ the adoption vector, indicating which technologies are adopted in $t=1$ at sites $i$ and $j$, and in $t=2$ at sites $i$ and $j$, respectively. If $x$ is the value of the technology $X_{i, t}$ that $i$ adopts, then the period $t$ utility of agent $i$ equals $U\left(X_{i, t}\right)=x+\lambda \hat{\pi}_{i, t}\left(\Omega_{i, t}\right)$, where $\hat{\pi}_{i, t}\left(\Omega_{i, t}\right)=\operatorname{Pr}\left(\theta_{i}=\bar{\theta} \mid \Omega_{i, t}\right)$ equals the belief that $i$ is highly able conditional on $\Omega_{i, t}$, and $\lambda>0$ is the relative weight of reputational concerns. We ignore time discounting. The center's utility equals the sum of the values of the technologies adopted in $t=2$.

Different decision processes cause differences in behaviour in the second period, but not in the first. This will be readily apparent from the analysis in the following sections. Independent of the decision process, period $t=1$ behaviour that maximizes agent $i$ 's utility is to follow his signal: $X_{i, 1}=Y$ if and only if $s_{i}=s^{Y}$. This maximizes the expected value of the technology and minimizes the probability of changing (or having to change) technology in period 2 .

An equilibrium consists of a communication strategy $\mu_{i}(\cdot)$ for each agent, a belief function $f_{i}(\cdot \mid I)$ for each decision maker, a decision strategy $d_{i}(\cdot)$ for each decision maker, and ex post reputations $\hat{\pi}_{i, t}(\cdot)$. We use the concept of Perfect Bayesian Equilibrium (from now on, equilibrium) to characterize behaviour. This requires (i) that the communication strategies are optimal for each type given decision makers' strategies and reputations; (ii) that the decision strategy is optimal given the belief functions and reputations; (iii) that beliefs and reputations are obtained using Bayes rule. Because of the inherent symmetry, we write the analysis from the point of view of agent $i=1$ and assume that $s_{1}=s^{Y}$. Of course, $s_{2} \in\left\{s^{Y}, s^{Z}\right\}$. We ignore babbling equilibria if an equilibrium in which information is transmitted exists. 


\section{Isolated agents}

Once agent 1 has followed his signal $s^{Y}$ in period 1 and observed value $y$, he has to decide whether to continue with his technology. Note that having received $s^{Y}$ and next observing $y$ allows an agent to update the expected value of the other technology,

$$
E\left[Z \mid s^{Y}, y\right]=\operatorname{Pr}\left(\bar{\theta} \mid s^{Y}, y\right) E\left[Z \mid s^{Y}, y, \bar{\theta}\right]+\operatorname{Pr}\left(\underline{\theta} \mid s^{Y}, y\right) E[Z]
$$

where we have used that $E\left[Z \mid s^{Y}, y, \underline{\theta}\right]=E[Z]$. Two effects of $y$ can be distinguished. First, the larger is $y$, the more likely it is that the agent is highly able and correctly identified the more valuable technology. This is the $\operatorname{Pr}\left(\bar{\theta} \mid s^{Y}, y\right)$ term. Second, conditional on the agent being highly able, a higher value of $y$ increases the expected value of $Z$. This is the $E\left[Z \mid s^{Y}, y, \bar{\theta}\right]$ term. Of course, $E\left[Z \mid s^{Y}, y, \bar{\theta}\right] \leq E[Z]$. The following lemma summarizes some characteristics of $E\left[Z \mid s^{Y}, y\right]$.

Lemma 1 The expected value of $Z$ given $s_{i}=s^{Y}$ and $y$ satisfies: (a) $E\left[Z \mid s^{Y}, 0\right]=$ $E\left[Z \mid s^{Y}, 1\right]=E[Z]$, and $E\left[Z \mid s^{Y}, y\right]<E[Z]$ for $y \in(0,1) ;(b) E\left[Z \mid s^{Y}, y\right]$ is decreasing in $y$ for $y<E\left[Z \mid s^{Y}, y\right]$, increasing for $y>E\left[Z \mid s^{Y}, y\right]$, and $y=E\left[Z \mid s^{Y}, y\right]$ has a unique solution.

This lemma is illustrated in Figure 1, panel a. The horizontal line represents the unconditional expectation $E[Z]$, and the conditional expectation $E\left[Z \mid s^{Y}, y\right]$ is a convex function of $y$.

Ignore reputational concerns for the moment. Given $I_{1}^{\text {ia }}=\left\{s^{Y}, y\right\}$, the decision strategy that maximizes the expected value of the technology adopted at site 1 in the second period, the first-best strategy, is to stick to the existing technology if and only if $y \geq E\left[Z \mid s^{Y}, y\right]$. It follows from lemma 1, part (b), and it is clear from Figure 1, panel a, that the first-best decision strategy is a single-threshold strategy,

$$
d_{1}^{\text {ia }}\left(I^{\mathrm{ia}} ; \bar{t}\right)= \begin{cases}Y & \text { if } y \geq \bar{t} \\ Z & \text { otherwise }\end{cases}
$$

with $\bar{t}=\bar{y}_{\text {ia }}^{F B}$ and where $\bar{y}_{\text {ia }}^{F B}$ solves $\bar{y}_{\text {ia }}^{F B}=E\left[Z \mid s^{Y}, \bar{y}_{\text {ia }}^{F B}\right]$. 
Figure 1: Isolated Agents. Panel a depicts the first-best threshold value; panel b the equilibrium threshold value $\bar{y}_{\text {ia }}^{*}$ for $\lambda<\bar{\lambda}_{\text {ia }}$; panel c reports the equilibrium values for $f_{X}=1$ and $\pi=1 / 2$. Thus, $\bar{\lambda}_{\text {ia }}=1$. Note that $\Delta \hat{\pi}^{*}$ is the equilibrium reputational gap.

(a) First-best

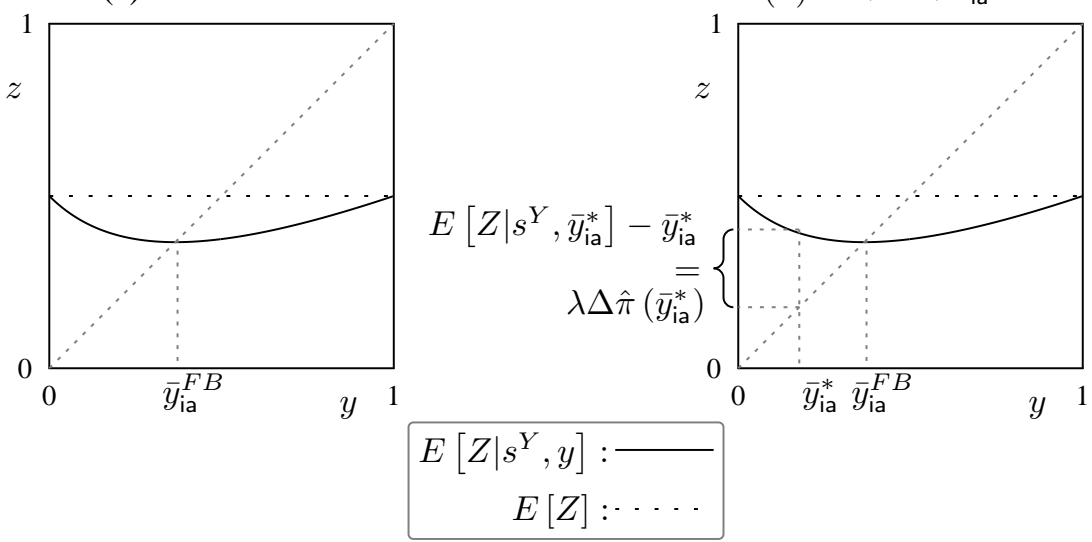

(c) $f_{X}=1$ and $\pi=1 / 2$

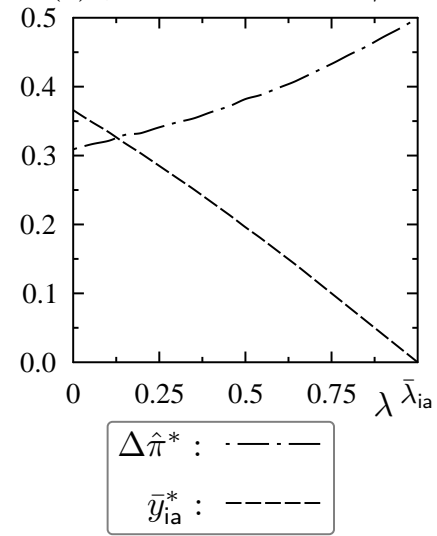

Besides being interested in picking the most valuable technology, an agent is also interested in his reputation. Consider a threshold decision strategy and any threshold value $\bar{t} \in(0,1)$. In case of isolated agents, markets only have local knowledge. Let $\hat{\pi}\left(Y, X_{1,2} ; \bar{t}\right)$ denote the reputation, obtained using Bayes' rule, if $X_{1,2} \in\{Y, Z\}$, and the agent uses the threshold $\bar{t}$. Then, ${ }^{21}$

$$
\hat{\pi}_{1}(Y, Y ; \bar{t})=\frac{1+F(\bar{t})}{1+F(\bar{t}) \pi} \pi>\pi>\hat{\pi}_{1}(Y, Z ; \bar{t})=\frac{F(\bar{t})}{F(\bar{t}) \pi+(1-\pi)} \pi .
$$

Irrespective of $\bar{t}$, continuation commands a higher reputation than switching to the other technology. Continuation suggests having observed a sufficiently high value of $y$. A highly able agent is more likely to have implemented a technology that generates a high value than a less able agent. Hence, as an agent cares about his reputation, he wants to deviate from the first-best decision rule by lowering the hurdle that his initial technology should pass for its continuation. The agent wants to give up technological adequacy for reputational benefits. We will call the difference $\hat{\pi}_{1}(Y, Y ; \bar{t})-\hat{\pi}_{1}(Y, Z ; \bar{t})$ the reputational gap. It is the source of the distortion. Proposition 1 describes equilibrium behaviour of an isolated agent.

Proposition 1 In case of isolated agents, and for $\lambda<\bar{\lambda}_{\mathrm{ia}}=E[Z] / \pi$, there exists an equilibrium in which the decision strategy is a single-threshold strategy with threshold value $\bar{y}_{\mathrm{i} a}^{*}$

\footnotetext{
${ }^{21}$ Deriviations can be found in the proof of Proposition 1 in the Appendix.
} 
that satisfies

$$
\lambda\left[\hat{\pi}_{1}\left(Y, Y ; \bar{y}_{\mathrm{ia}}^{*}\right)-\hat{\pi}_{1}\left(Y, Z ; \bar{y}_{\mathrm{ia}}^{*}\right)\right]=E\left[Z \mid s^{Y}, \bar{y}_{\mathrm{ia}}^{*}\right]-\bar{y}_{\mathrm{ia}}^{*},
$$

with $\bar{y}_{\mathrm{ia}}^{*} \in\left(0, \bar{y}_{\mathrm{ia}}^{F B}\right) . \bar{y}_{\mathrm{ia}}^{*}$ is a decreasing function of $\lambda .^{22}$ For $\lambda \geq \bar{\lambda}_{\mathrm{ia}}, \bar{y}_{\mathrm{ia}}^{*}=0$, i.e., agent 1 always continues his initial technology, and $\hat{\pi}_{1}(Y, Y ; 0)=\pi$ and $\hat{\pi}_{1}(Y, Z ; 0)=0$.

Eq (3) is illustrated in Figure 1, panel b. At the threshold value $\bar{y}_{\mathrm{i}}^{*}$ the agent is indifferent between sticking to $Y$ and switching to $Z$. This can also be seen by rewriting (3) as $\bar{y}_{\mathrm{ia}}^{*}+\lambda \hat{\pi}_{1}\left(Y, Y ; \bar{y}_{\mathrm{ia}}^{*}\right)=E\left[Z \mid s^{Y}, \bar{y}_{\mathrm{ia}}^{*}\right]+\lambda \hat{\pi}_{1}\left(Y, Z ; \bar{y}_{\mathrm{ia}}^{*}\right)$. The left-hand side equals the value of continuing with $Y$ if its observed value equals $\bar{y}_{\text {ia }}^{*}$, whereas the left-hand side equals the value of switching technology for the same observed value of $Y$. It follows from (2) that the lower $\bar{y}_{\mathrm{ia}}^{*}$ is, the lower is the reputation the agent commands in case of sticking to the original technology and in case of switching technologies. If the hurdle for continuation is lowered, passing the hurdle becomes a less convincing signal of ability. At the same time, not passing a lower hurdle becomes a stronger signal of incompetence. It can be checked that the reputational gap increases the lower is $\bar{y}_{\mathrm{ia}}^{*}$. As the reputational gap is still strictly positive for a threshold value equal to zero, it follows from (3) that for $\lambda \geq \bar{\lambda}_{\mathrm{ia}} \bar{y}_{\mathrm{ia}}^{*}=0$ : the agent will continue with his initial choice of technology irrespective of its observed value. Figure 1, panel c illustrates the proposition for a uniform distribution and $\pi=1 / 2$. It shows the equilibrium values of $\bar{y}_{\mathrm{i} a}^{*}$ and $\hat{\pi}_{1}\left(Y, Y ; \bar{y}_{\mathrm{ia}}^{*}\right)-\hat{\pi}_{1}\left(Y, Z ; \bar{y}_{\mathrm{ia}}^{*}\right)$.

\section{$5 \quad$ Decentralized learning}

In this section, we assume that the right to decide about the technology to be adopted in period two remains with the agents. We begin by describing first-best behaviour in a decentralized process. In the communication stage each agent truthfully reveals his private information. Say that 1 truthfully reveals his private information if, for all $y \in[0,1]$, and all $X_{2,1} \in\{Y, Z\}, \operatorname{Pr}\left(m_{1} \mid s^{Y}, y, X_{2,1}\right)=1$ if $m_{1}=y$ and $\operatorname{Pr}\left(m_{1} \mid s^{Y}, y, X_{2,1}\right)=0$ otherwise.

\footnotetext{
${ }^{22}$ We cannot exclude the possibility of multiple equilibria in general. In case of multiple equilibria, we show that the highest and the lowest equilibrium values of $\bar{y}_{\text {ia }}^{*}$ are decreasing functions of $\lambda$. We have established numerically that in case of the uniform distribution, the equilibrium is unique, in this and all other sections.
} 
Next, the first-best decision strategy equals

$$
d_{1}^{\mathrm{dl}}\left(I_{1}^{\mathrm{dl}} ; \bar{y}_{\mathrm{S}}^{F B}\right)= \begin{cases}Y & \text { if } X_{2,1}=Y \text { and } y \geq \bar{y}_{\mathrm{S}}^{F B} \\ Y & \text { if } X_{2,1}=Z \text { and } y \geq z \\ Z & \text { otherwise }\end{cases}
$$

where $\bar{y}_{\mathrm{S}}^{F B}$ satisfies $\bar{y}_{\mathrm{S}}^{F B}=E\left[Z \mid s^{Y}, s^{Y}, \bar{y}_{\mathrm{S}}^{F B}\right]$. That is, if both agents adopted the same technology, each agent should continue this technology if its value is larger than $\bar{y}_{\mathrm{S}}^{F B} .^{23}$ If instead agents adopted different technologies, they should next choose the one with superior performance. In 5.1 we study equilibrium behaviour in case reputations are locally determined, and in 5.2 we turn to reputations that are globally determined. In 7.1, we compare the performance of decentralized learning under both types of reputation formation.

\subsection{Locally determined reputations}

Can truthful revelation be part of an equilibrium? With agent 1's reputation independent of what the other agent decides, and with agent 1 being free to choose what technology to adopt in $t=2$, truthful revelation of the technology's value is an equilibrium communication strategy for each agent ${ }^{24}$. Absent any motive to influence the other agent, the quality of the information exchange is high.

Once communication has taken place, each agent independently decides whether to continue with his original technology or to switch to the other technology. Let a double-threshold strategy $d_{1}^{\mathrm{dl}}\left(I_{1}^{\mathrm{dl}} ; \bar{t}_{\mathrm{S}}, \bar{t}_{\mathrm{D}}\right)$ with thresholds $\left(\bar{t}_{\mathrm{S}}, \bar{t}_{\mathrm{D}}\right) \geqq 0$ be defined as

$$
d_{1}^{\mathrm{dl}}\left(I_{1}^{\mathrm{dl}} ; \bar{t}_{\mathrm{S}}, \bar{t}_{\mathrm{D}}\right)= \begin{cases}Y & \text { if } X_{2,1}=Y \text { and } y \geq \bar{t}_{\mathrm{S}} \\ Y & \text { if } X_{2,1}=Z \text { and } y \geq m_{2}-\bar{t}_{\mathrm{D}} \\ Z & \text { otherwise }\end{cases}
$$

That is, agent 1 continues with his original technology $Y$ (i) if both agents used the same technology and its value exceeds $\bar{t}_{\mathrm{S}}$; or (ii) if the agents used different technologies, but the

\footnotetext{
${ }^{23}$ Of course, the fact that both experts used the same technology in the first period bodes well for the superiority of this technology: $\bar{y}_{\mathrm{S}}^{F B}<\bar{y}_{\mathrm{ia}}^{F B}$.

${ }^{24}$ In fact, it is payoff irrelevant, both directly (cheap talk) and indirectly, as 2's actions do not affect 1's payoff.
} 
other technology is either less valuable or its superior performance does not exceed by a margin larger than $\bar{t}_{\mathrm{D}}$ the value of the current technology. Let $\hat{\pi}_{1}\left(Y, X ; \bar{t}_{\mathrm{S}}, \bar{t}_{\mathrm{D}}\right)$ denote 1's reputation if he uses the double-threshold strategy, and adopts $X_{1,2}=X \in\{Y, Z\}$ in period 2.

To see that an agent wants to distort the decision on $X_{1,2}$, suppose 1 were to use the first-best threshold values, $\left(\bar{t}_{\mathrm{S}}, \bar{t}_{\mathrm{D}}\right)=\left(\bar{y}_{\mathrm{dl}}^{F B}, 0\right)$. If 1 continues with his initial technology, the market deduces that either the same technology was used at the other site and its observed value exceeded $\bar{y}_{\mathrm{dl}}^{F B}$, or that the other site used the other technology which proved to be of inferior quality. Either event strengthens 1's reputation. Analogously, discontinuing a technology hurts a reputation. As a result, reputational concerns induce an agent to distort the decision in $t=2$. If both agents adopted $Y$ in $t=1$, then agent 1 sticks to this technology if and only if $y+\lambda \hat{\pi}_{1}\left(Y, Y ; \bar{t}_{\mathrm{S}}, \bar{t}_{\mathrm{D}}\right) \geq E\left[Z \mid s^{Y}, s^{Y}, y\right]+\lambda \hat{\pi}_{1}\left(Y, Z ; \bar{t}_{\mathrm{S}}, \bar{t}_{\mathrm{D}}\right)$. Similarly, in case agents adopted different technologies, agent 1 wants to continue with $Y$ iff $y+\lambda \hat{\pi}_{1}\left(Y, Y ; \bar{t}_{\mathrm{S}}, \bar{t}_{\mathrm{D}}\right) \geq z+\lambda \hat{\pi}_{1}\left(Y, Z ; \bar{t}_{\mathrm{S}}, \bar{t}_{\mathrm{D}}\right)$. Proposition 2 describes equilibrium behaviour. Note that lo stands for locally determined reputations.

Proposition 2 Define $\underline{\lambda}_{\mathrm{dl}}^{\mathrm{lo}}=E[Z] / \hat{\pi}_{1}(Y, Y ; 0, E[Z])$ and $\bar{\lambda}_{\mathrm{dl}}^{\mathrm{lo}}=1 / \pi$. In case of decentralized learning and locally determined reputations, an equilibrium exists in which

(i) truthful revelation is the communication strategy;

(ii) the belief functions are $\operatorname{Pr}\left(x_{2,1} \mid m_{2}\right)=1$ for $x_{2,1}=m_{2}$ and $\operatorname{Pr}\left(x_{2,1} \mid m_{2}\right)=0$ for $x_{2,1} \neq m_{2}$; (iii) the decision strategy is a double-threshold strategy. For $\lambda<\underline{\lambda}_{\mathrm{dl}}^{\mathrm{lo}}$, threshold values $\left(\bar{t}_{\mathrm{S}}^{*}, \bar{t}_{\mathrm{D}}^{*}\right)$ satisfy

$$
\begin{aligned}
\lambda\left[\hat{\pi}_{1}\left(Y, Y ; \bar{t}_{\mathrm{S}}^{*}, \bar{t}_{\mathrm{D}}^{*}\right)-\hat{\pi}_{1}\left(Y, Z ; \bar{t}_{\mathrm{S}}^{*}, \bar{t}_{\mathrm{D}}^{*}\right)\right] & =E\left[Z \mid s^{Y}, s^{Y}, \bar{t}_{\mathrm{S}}^{*}\right]-\bar{t}_{\mathrm{S}}^{*} \\
\lambda\left[\hat{\pi}_{1}\left(Y, Y ; \bar{t}_{\mathrm{S}}^{*}, \bar{t}_{\mathrm{D}}^{*}\right)-\hat{\pi}_{1}\left(Y, Z ; \bar{t}_{\mathrm{S}}^{*}, \bar{t}_{\mathrm{D}}^{*}\right)\right] & =\bar{t}_{\mathrm{D}}^{*},
\end{aligned}
$$

with $\bar{t}_{\mathrm{S}}^{*} \in\left(0, \bar{y}_{\mathrm{S}}^{F B}\right)$ and $\bar{t}_{\mathrm{D}}^{*} \in(0,1)$. For $\lambda \in\left[\underline{\lambda}_{\mathrm{dl}}^{\mathrm{lo}}, \bar{\lambda}_{\mathrm{dl}}^{\mathrm{lo}}\right)$, threshold values are $\left(0, \bar{t}_{\mathrm{D}}^{*}\right)$ and $\bar{t}_{\mathrm{D}}^{*}$ solves $\lambda \hat{\pi}_{1}\left(Y, Y ; 0, \bar{t}_{\mathrm{D}}^{*}\right)=\bar{t}_{\mathrm{D}}^{*}$. Finally, for $\lambda \geq \bar{\lambda}_{\mathrm{dl}}^{\text {lo }}$, threshold values equal $(0,1)$.

Figure 2, panels a and b show the structure of the equilibrium. For $\lambda<\underline{\lambda}_{\mathrm{dl}}^{\mathrm{lo}}$, see panel a and Eqs (4) and (5), in equilibrium the size of the distortions, $E\left[Z \mid s^{Y}, s^{Y}, \bar{t}_{\mathrm{S}}^{*}\right]-\bar{t}_{\mathrm{S}}^{*}$ and $\bar{t}_{\mathrm{D}}^{*}$, and the value of the reputational gap, $\lambda\left[\hat{\pi}_{1}\left(Y, Y ; \bar{t}_{\mathrm{S}}^{*}, \bar{t}_{\mathrm{D}}^{*}\right)-\hat{\pi}_{1}\left(Y, Z ; \bar{t}_{\mathrm{S}}^{*}, \bar{t}_{\mathrm{D}}^{*}\right)\right]$, are the same. 
The loss in technological value due to the distortion should in either case be compensated by the same boost in reputation. At $\lambda=\underline{\lambda}_{\mathrm{d}}^{\mathrm{lo}}, \bar{t}_{\mathrm{S}}^{*}=0$, and $\bar{t}_{\mathrm{D}}^{*}=E[Z]$. Then, the market infers from observing $(Y, Z)$ that agents initially used different technologies and $y<z$, and so 1 initially picked the inferior technology, $\hat{\pi}_{1}(Y, Z ; 0, E[Z])=0$. Also, $\hat{\pi}_{1}(Y, Y ; 0, E[Z])>\pi$ as the market infers from $(Y, Y)$ that either both agents initially received $s^{Y}$, or that the other agent received $s^{Z}$ but $y \geq z-\bar{t}_{\mathrm{D}}^{*}$. Either possibility boosts agent 1's reputation. It follows from (4) that $\underline{d}_{\mathrm{dl}}^{\mathrm{lo}}=E[Z] / \hat{\pi}_{1}(Y, Y ; 0, E[Z])<E[Z] / \pi$.

(a) $\lambda<\underline{\lambda}_{\mathrm{dl}}^{\text {lo }}$

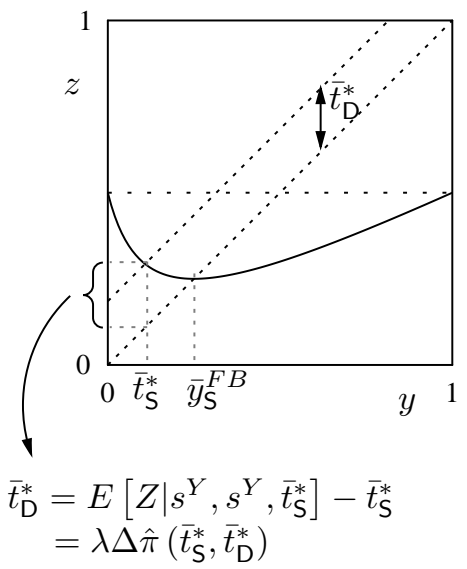

(b) $\lambda \in\left(\underline{\lambda}_{\mathrm{dl}}^{\mathrm{lo}}, \bar{\lambda}_{\mathrm{dl}}^{\mathrm{lo}}\right)$

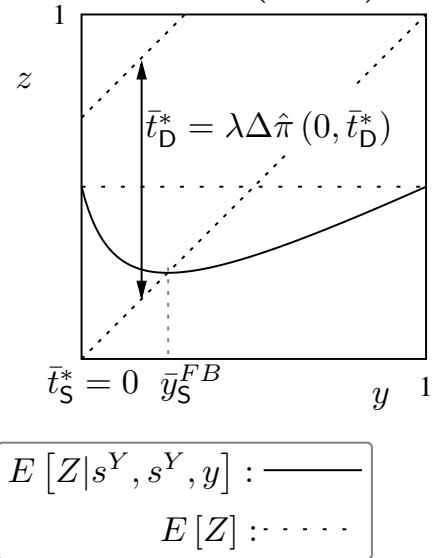

(c) $f_{X}=1$ and $\pi=1 / 2$

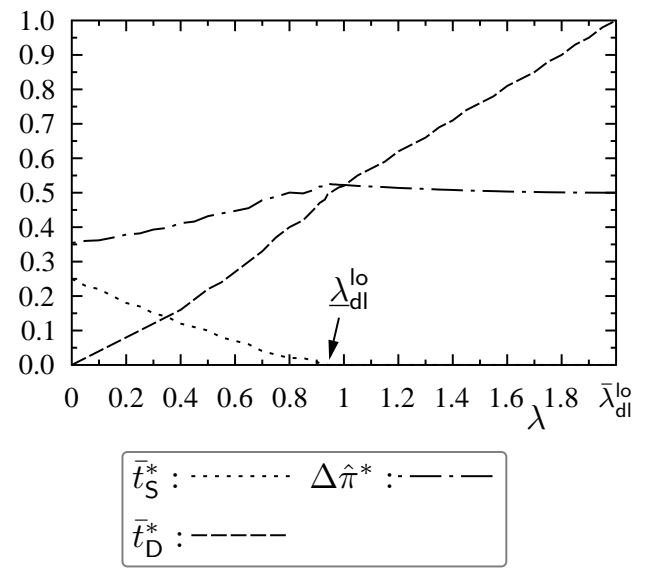

Figure 2: Decentralized learning and locally determined reputations. Panels a and b depict the structure of equilibrium. Panel c reports equilibrium threshold values and the reputational gap for the uniform distribution and $\pi=1 / 2$. Hence, $\underline{d}_{\mathrm{dl}}^{\mathrm{lo}}<1$ and $\bar{\lambda}_{\mathrm{dl}}^{\mathrm{lo}}=2$.

For $\lambda \in\left[\underline{\lambda}_{\mathrm{dl}}^{\text {lo }}, \bar{\lambda}_{\mathrm{dl}}^{\text {lo }}\right)$, illustrated in panel b, if 1 learns that 2 used the same technology, he continues his initial technology irrespective of its value $y, \bar{t}_{\mathrm{S}}^{*}=0$, whereas if 1 learns that 2 used a different technology, 1 may still change technology. For $\lambda \geq \bar{\lambda}_{\mathrm{dl}}^{\mathrm{lo}}, 1$ sticks to his initial technology $Y$, irrespective of its value $y$, and regardless of what 2 reports, $\left(\bar{t}_{\mathrm{S}}^{*}, \bar{t}_{\mathrm{D}}^{*}\right)=(0,1)$. Then $\hat{\pi}_{1}(Y, Y ; 0,1)=\pi$ as continuation of $Y$ does not reveal any information on ability, while $\hat{\pi}_{1}(Y, Z ; 0,1)=0$ is a plausible out-of-equilibrium belief. Hence, $\bar{\lambda}_{\mathrm{dl}}^{\mathrm{lo}}=1 / \pi$. Panel c illustrates the reputational gap and the threshold values for the uniform distribution and $\pi=1 / 2$. The reputational gap rises for $\lambda<\underline{\lambda}_{\mathrm{dl}}^{\text {lo }}$ to $\hat{\pi}_{1}(Y, Y ; 0, E[Z])>\pi$, and declines to $\pi$ for $\underline{d}_{\mathrm{dl}}^{\mathrm{lo}}<\lambda \leq \bar{\lambda}_{\mathrm{dl}}^{\mathrm{lo}}$. 


\subsection{Globally determined reputations}

We start by showing that first-best behaviour, described on page 17, is not equilibrium behaviour. Suppose imputed equilibrium behaviour is first-best behaviour. Then, if agents initially adopted different technologies, the only adoption vectors possible are $(Y, Z, Y, Y)$ and $(Y, Z, Z, Z)$. The inference the market draws from the first (resp. second) vector is that $Y$ (resp. $Z$ ) is the superior technology, and that 1 made the correct (resp. wrong) choice. The correct choice can be thanks to skill, or due to low ability and luck. The wrong choice, by contrast, must be due to low ability. Hence ${ }^{25}, \hat{\pi}_{1}(Y, Z, Y, Y)=\frac{2 \pi}{1+\pi}>\pi$ and $\hat{\pi}_{1}(Y, Z, Z, Z)=0$. Clearly, from a reputational point of view, the former is the best and the latter is the worst that could happen to agent 1 . Could 1 convince 2 to adopt "his" technology? Rather than truthful revelation, consider the following unilateral deviation strategy in case of different initial technologies: "send $m_{1}=1$ independent of $y$, and in the decision stage stick to $Y$ if and only if $y \geq m_{2}$." The effect of this deviation strategy is that 1 convinces 2 to adopt $Y$ in $t=2$. Whether 1 continues with $Y$ depends on the reported value $m_{2}$ and $y$. For $y \geq m_{2}$, the adoption vector in $t=2$ becomes $(Y, Z, Y, Y)$, the same as it would have been had 1 stuck to truthful revelation. If $y<m_{2}$, the adoption vector in case of the deviation strategy equals $(Y, Z, Z, Y)$, whereas in case of truthful revelation it would have been $(Y, Z, Z, Z)$. The reputation implied by such a deviation is not determined by the imputed equilibrium behaviour. However, it is consistent with the model to assume that, given any adoption vector, any increase in the use at $t=2$ of the technology 1 adopted in $t=1$ does not decreases the reputation of 1 .

Assumption 1 Consider any adoption vector with $X_{1,1}=Y$. The reputation of 1 does not decrease if 1 (resp. 2) changes from $X_{1,2}=Z$ to $X_{1,2}=Y$ (resp. from $X_{2,2}=Z$ to $\left.X_{2,2}=Y\right)$.

With this assumption, the deviation is advantageous in terms of reputation, and costless in terms of technical adequacy. We have proved the next Lemma.

Lemma 2 First-best behaviour is not equilibrium behaviour in case of decentralized learning with globally determined reputations.

\footnotetext{
${ }^{25}$ See the proof of Proposition 3.
} 
The above line of reasoning can be applied to any imputed equilibrium in which, in case agents started by adopting different technologies, 2's decision regarding $X_{2,2}$ depends on the message $m_{1}$ of 1 . The profitable deviation is then for 1 to send the message that induces 2 to adopt $Y$, and to continue to base his own decision for $t=2$ on a comparison of $y$ and the expected value of $Z$ given $m_{2}$. This shows that the unique equilibrium communication strategy in case $X_{2,1}=Z$ is a pooling strategy. ${ }^{26}$ The interest an agent has to convince the other to agent to switch technology destroys all meaningful communication. This is in line with one of the concerns expressed about the OMC in the EU, a case of a decentralized learning process with globally determined reputations.

In case agents initially adopted the same technology, $Y$, it is easy to see that truthful revelation is an equilibrium strategy. Communication is also irrelevant. ${ }^{27}$ Proposition 3 below establishes that in this case an agent wants to deviate from first-best behaviour in the decision stage.

As communication breaks down in case of different initial technologies, and is irrelevant in case of the same initial technology, the equilibrium decision strategy of 1 amounts to a comparison of $y$ with a cut-off value that depends on the number of agents that used the same technology in $t=1$. Let a double-cut-off strategy with cut-offs $\left(\bar{c}_{S}, \bar{c}_{\mathrm{D}}\right) \geqq 0$ be defined as

$$
d_{1}^{\mathrm{dl}}\left(I_{1}^{\mathrm{dl}} ; \bar{c}_{\mathrm{S}}, \bar{c}_{\mathrm{D}}\right)= \begin{cases}Y & \text { if } X_{2,1}=Y \text { and } y \geq \bar{c}_{\mathrm{S}} \\ Y & \text { if } X_{2,1}=Z \text { and } y \geq \bar{c}_{\mathrm{D}} \\ Z & \text { otherwise. }\end{cases}
$$

Of course, conditional on the information exchanged, the values of $\bar{c}_{S}$ and $\bar{c}_{\mathrm{D}}$ that would maximize the technological value are $\bar{c}_{\mathrm{S}}=\bar{y}_{\mathrm{S}}^{F B}$, and $\bar{c}_{\mathrm{D}}=E[Z] .^{28}$ The next Proposition describes equilibrium behaviour. Note that gl stands for globally determined reputations. ${ }^{29}$

\footnotetext{
${ }^{26}$ To avoid a discussion of out-of-equilibrium beliefs, we assume that each agent uses a probability distribution over the full support $[0,1]$ that is independent of the value $x$ he observed. We refer to this equilibrium communication strategy simply by "pooling strategy".

${ }^{27}$ This is so as in our model technologies have a common value that is learned before agents communicate in $t=2$.

${ }^{28}$ Note that $E\left[Z \mid s^{Y}, s^{Z}, y\right]=E[Z]$.

${ }^{29}$ In what follows, we assume that the out-of-equilibrium belief $\hat{\pi}_{1}(Y, Y, Z, Y)$ equals $\hat{\pi}_{1}(Y, Y, Z, Z)$.
} 
Proposition 3 Define $\underline{\mathrm{dl}}_{\mathrm{gl}}^{\mathrm{gl}}=E[Z] \frac{1+\pi^{2}}{\pi(1+\pi)}$ and $\bar{\lambda}_{\mathrm{dl}}^{\mathrm{gl}}=E[Z] \frac{1+\pi}{\pi}$. In case of decentralized learning with globally determined reputations, there exists an equilibrium in which

(i) the communication strategy is (a) a pooling strategy if initial technologies differ, and (b) truthful revelation if initial technologies are the same;

(ii) the belief function equals (a) the density $f_{1}\left(z \mid I_{1}^{\mathrm{dl}}\right)=f(z)$ for all $z$ and $m_{2}$ in case $X_{2,1}=Z$; and (b) discrete probabilities in case $X_{2,1}=Y, \operatorname{Pr}\left(y \mid m_{2}\right)=1$ for $y=m_{2}$ and $\operatorname{Pr}\left(y \mid m_{2}\right)=0$ for $y \neq m_{2}$;

(iii) the decision strategy is a double-cut-off strategy. The cut-off value in case initial technologies are the same, $\bar{c}_{\mathrm{S}}^{*}$, satisfies

$$
\lambda\left[\hat{\pi}_{1}\left(Y, Y, Y, Y ; \bar{c}_{\mathrm{S}}^{*}\right)-\hat{\pi}_{1}(Y, Y, Z, Y)\right]=E\left[Z \mid s^{Y}, s^{Y}, \bar{c}_{\mathrm{S}}^{*}\right]-\bar{c}_{\mathrm{S}}^{*},
$$

with $\bar{c}_{\mathrm{S}}^{*} \in\left(0, \bar{y}_{\mathrm{S}}^{F B}\right)$ for $\lambda<\underline{\lambda}_{\mathrm{dl}}^{\mathrm{gl}}$. $\bar{c}_{\mathrm{S}}^{*}$ is a decreasing function of $\lambda .^{30}$ For $\lambda \geq \underline{\lambda}_{\mathrm{dl}}^{\mathrm{gl}}$, $\bar{c}_{\mathrm{S}}^{*}=0$. The cut-off value in case initial technologies differ, $\bar{c}_{\mathrm{D}}^{*}$, satisfies

$$
\lambda \frac{\pi}{1+\pi}=E[Z]-\bar{c}_{\mathrm{D}}^{*}
$$

with $\bar{c}_{\mathrm{D}}^{*} \in\left(0, \bar{y}_{\mathrm{D}}^{F B}\right)$ for $\lambda<\bar{\lambda}_{\mathrm{dl}}^{\mathrm{gl}}$. $\bar{c}_{\mathrm{D}}^{*}$ is a decreasing function of $\lambda$. For $\lambda \geq \bar{\lambda}_{\mathrm{dl}}^{\mathrm{gl}}, \bar{c}_{\mathrm{D}}^{*}=0$.

Figure 3, panels a and b correspond to (6) and (7), respectively.

Panel $\mathrm{c}$ shows the equilibrium values in case of $f_{X}=1$ and $\pi=1 / 2$. Eq (7) shows that if agents adopted different technologies in $t=1$, then the reputational gap is a constant function of $\bar{c}_{\mathrm{D}}^{*}$. To understand why, recall that ability means the ability to identify the better technology. When the market observes that agents initially used different technologies, the agents' choices in $t=2$ either allow the market to infer who used the better and the worse technology (i.c., $(Y, Z, Z, Z)$ and $(Y, Z, Y, Y))$ or does not allow the market to infer any information on the relative performance of the technologies (i.c., $(Y, Z, Y, Z)$ and $(Y, Z, Z, Y))$. The value of $\bar{c}_{\mathrm{D}}^{*}$ does not provide additional information on an agent's ability. Of course, if the market observes that agents initially adopted the same technology, $\hat{\pi}_{1}\left(Y, Y, Y, Y ; \bar{c}_{\mathrm{S}}^{*}\right)$ does depend on the cut-off value: the lower is $\bar{c}_{\mathrm{S}}^{*}$, the lower is the reputation an agent commands in case of continuation.

\footnotetext{
${ }^{30}$ The remark made in footnote 22 applies.
} 
Figure 3: Decentralized learning and globally determined reputations. Panels a and b depict the structure of equilibrium. Panel c reports equilibrium cut-off values and reputational gaps for $f_{X}=1$ and $\pi=1 / 2 . \Delta \hat{\pi}_{1}(\mathrm{~S})$ denotes $\hat{\pi}_{1}\left(Y, Y, Y, Y ; \bar{c}_{S}^{*}\right)-\hat{\pi}_{1}(Y, Y, Z, Y)$, and $\Delta \hat{\pi}_{1}(\mathrm{D})=\frac{\pi}{1+\pi}$.
(a) $X_{j, 1}=Y$
(b) $X_{j, 1}=Z$
(c) $f_{X}=1$ and $\pi=1 / 2$
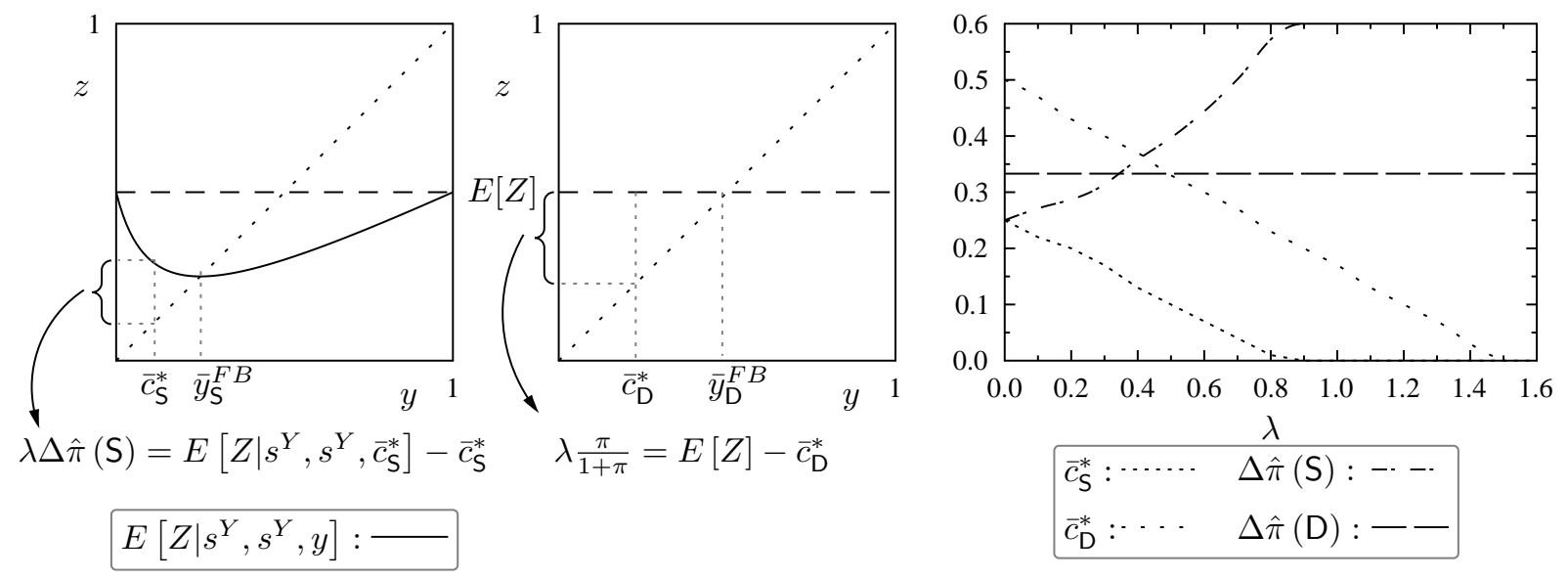

In case reputations are globally determined, there are two reputational gaps. In panel c, $\Delta \hat{\pi}_{1}(\mathrm{~S})$ is the reputational gap in case both agents started with $Y$. Starting with the same technology is a good sign for each agent's ability. Thus, even if $\lambda$ increases and $\bar{c}_{\mathrm{S}}^{*}$ goes to zero $\hat{\pi}_{1}\left(Y, Y, Y, Y ; \overline{\mathrm{s}}_{\mathrm{S}}^{*}\right)>\pi$. On the other hand, the lower is $\bar{c}_{\mathrm{S}}^{*}$, the more switching indicates a poor choice in period 1 . The net effect is that the gap increases in $\lambda$, see the proof. In case reputations are locally determined, there is a single reputational gap, see the left-hand sides of (4) and (5) in Proposition 2.

\section{Centralized learning}

Now we turn to the case where the local agents who gained experience in period one do not have the right to decide on the technology to be adopted in period two; instead, the "centre" decides. First-best behaviour in the case of a centralized learning process is for each agent to truthfully reveal his private information, and for the center next to pick the technology 
with the higher, reported or expected, value:

$$
d_{C}\left(I_{C} ; \bar{y}_{\mathrm{S}}^{F B}\right)= \begin{cases}Y, Y & \text { if } X_{2,1}=Y \text { and } m_{1} \geq \bar{y}_{\mathrm{S}}^{F B} \\ Z, Z & \text { if } X_{2,1}=Y \text { and } m_{1}<\bar{y}_{\mathrm{S}}^{F B} \\ Y, Y & \text { if } X_{2,1}=Z \text { and } m_{1}>m_{2} \\ Z, Z & \text { otherwise. }\end{cases}
$$

We start by showing that first-best behaviour is not equilibrium behaviour in case of centralized learning.

Lemma 3 Under centralized learning, an equilibrium in which agents truthfully reveal their private information does not exist, neither in case of locally nor in case of globally determined reputations.

It suffices to show that agent 1 has an incentive to slightly exaggerate the value of $Y$ in case $j$ adopted a different solution. If agents and planner were to stick to first-best behaviour, then an agent commands a higher reputation if he is allowed to continue with "his" solution than if he is forced to change. If reputations are determined locally, $\hat{\pi}_{1}(Y, Y)>\hat{\pi}_{1}(Y, Z)$, while if they are determined globally $\hat{\pi}_{i}(Y, Z, Y, Y)>\hat{\pi}_{i}(Y, Z, Z, Z)$. In either case, assume $i$ deviates by communicating a slightly exaggerated value of his technology, $y+\varepsilon>y$ instead of $y$, with $\varepsilon>0$. Conditional on this exaggeration changing the planner's decision i.e., for $z \in(y, y+\varepsilon)$, the benefits equal $\lambda\left[\hat{\pi}_{i}(Y, Z, Y, Y)-\hat{\pi}_{i}(Y, Z, Z, Z)\right]>0$ and are independent of $\varepsilon$, whereas the costs can be made arbitrarily small by reducing the value of $\varepsilon$. This shows that a profitable deviation from first-best behaviour exists.

Of course, in equilibrium an agent cannot "systematically exaggerate" as then the center could simply undo the exaggeration. Instead, as in Crawford and Sobel (1982), in equilibrium information is lost as the agent adds noise to his message: he partitions the space of possible technology values $[0,1]$ into intervals, and reports only to which interval the value of his technology belongs. That is, he ranks its value, and the number of intervals equals the number of possible ranks.

Let $\mathbf{a}(N) \equiv\left(a_{0}(N), \ldots, a_{N}(N)\right)$ denote a partition of $[0,1]$ in $N$ intervals, with $0=$ $a_{0}(N)<a_{1}(N)<\cdots<a_{N}(N)=1$. Agent 1 is said to use a partition strategy to communicate if there exists a tuple $(N, \mathbf{a}(N))$, such that $\mu_{1}^{\mathrm{p}}\left(m_{1} \mid s^{Y}, y, X_{2,1}\right)$ is uniform, 
supported on $\left[a_{r}(N), a_{r+1}(N)\right]$ if $y \in\left(a_{r}(N), a_{r+1}(N)\right)$ for $r=0, \ldots, N-1$. $^{31}$ We focus on the highest value of $N$ consistent with incentives. Say that agent 1 sends influential information (or that communication is influential) if there are two messages $m_{1}$ and $m_{1}^{\prime}$ about $Y$ and a message $m_{2}$ about $Z$ such that $d_{C}\left(m_{1}, m_{2}\right)=Y$ with probability one and $d_{C}\left(m_{1}^{\prime}, m_{2}\right)=Z$ with probability smaller than one. That is, the agent uses at least two ranks, $N \geq 2$. To save space, we write a instead of $\mathbf{a}(N)$ if this does not lead to confusion.

Does an agent truthfully report the value of his technology to the center if the other agent uses the same technology in $t=1$ ? Agent $i$ 's interest are different from those of the center, but identical to those of the agent $j$. This offers room for the agents to (tacitly) collude, and to induce the center to choose the technology they deem best. Each can send either of two messages, one such that the center will next decide that the technology is sufficiently good to merit continuation, and one inducing the center to force the agents to switch. Note that collusive behaviour of this sort seems easy to sustain as there is no asymmetric information among the agents. ${ }^{32}$ Although this is a partition strategy with $N \leq 2$, to distinguish it from the more general partition strategy in case agents use different technologies, we refer to it as a collusion strategy. It is completely characterized by a single value, $\bar{y}_{\mathrm{S}} \in[0,1]$, for which an agent is indifferent between sending one message rather than the other.

Let the center choose the technology that is the better one given the messages of the agents. In case they rank different technologies the same, the center is indifferent and tosses a coin. Even if both agents report on the same technology, the center may still decide to

\footnotetext{
${ }^{31}$ Note that between any two partitions the expert uses a random strategy. This guarantees that in equilibrium any possible message is sent with strictly positive probability. A discussion of out-of-equilibrium beliefs (what should the planner think about the value of a technology if he were to observe a non-equilibrium message?) can thus be avoided.

${ }^{32}$ In a previous version of this paper we show that truthfully revealing information to the center in case agents use the same technology can be part of equilibrium. However, it amounts to playing a weakly dominated strategy, an unlikely candidate to describe agents' behaviour.
} 
make them switch to the other technology. Formally,

$$
d_{C}\left(I_{C}\right)=\left\{\begin{array}{lll}
Y, Y & \text { if } X_{2,1}=Y & \text { and } E\left[Y \mid m_{\min }\right] \geq E\left[Z \mid m_{\min }\right] \\
Z, Z & \text { if } X_{2,1}=Y & \text { and } E\left[Y \mid m_{\min }\right]<E\left[Z \mid m_{\min }\right] \\
Y, Y & \text { if } X_{2,1}=Z & \text { and } E\left[Y \mid I_{C}\right]>E\left[Z \mid I_{C}\right] \\
Y, Y & \text { if } X_{2,1}=Z & \text { and } E\left[Y \mid I_{C}\right]=E\left[Z \mid I_{C}\right] \text { and coin }=Y \\
Z, Z & \text { otherwise, } &
\end{array}\right.
$$

where "coin $=Y$ " means that the center flips a fair coin with faces $Y$ and $Z$, and $Y$ comes up, and where $m_{\min }:=\min \left[m_{1}, m_{2}\right]$ is the lower valued message sent concerning the same technology. The contents of these messages - what they imply concerning the expected value of the technology - are the same if $m_{1}, m_{2} \in\left[a_{r-1}, a_{r}\right)$ and they differ if $m_{1}<a_{r} \leq$ $m_{2}$ for some $r{ }^{33}$ To state the belief function of the center, define a truncated density as follows: $\operatorname{Tr}\left(x ; a_{r}, a_{r+1}\right)=g(x) /\left(F\left(a_{r+1}\right)-F\left(a_{r}\right)\right)$, where $g(x)=f(x)$ for $x \in\left[a_{r}, a_{r+1}\right]$ and $g(x)=0$ everywhere else. The next proposition characterizes equilibrium behaviour.

Proposition 4 Define $\bar{\lambda}_{\mathrm{cl}}^{\mathrm{lo}}=E[Z] \frac{\left(3+\pi^{2}\right)(1+\pi)}{4 \pi^{2}}$ and $\bar{\lambda}_{\mathrm{cl}}^{\mathrm{gl}}=E[Z] \frac{1+\pi^{2}}{\pi(1+\pi)}$. In case of centralized learning, there exists an equilibrium in which

(i) the center's decision strategy is as defined in (8).

(ii) the communication strategy is (a) a partition strategy $\left(N^{*}, \mathbf{a}^{*}\right)$ if initial technologies differ, and (b) a collusion strategy $\bar{y}_{\mathrm{S}}^{*}$ if initial technologies are the same;

(ii) the center's belief function is (a) $f_{1}\left(x_{i, 1} \mid I_{C}\right)=\operatorname{Tr}\left(x_{i, 1} ; a_{r}^{*}, a_{r+1}^{*}\right)$ for $m_{1}^{X} \in\left(a_{r}^{*}, a_{r+1}^{*}\right)$ for $r=0, \ldots, N^{*}-1$ if initial technologies differ, and (b) $f_{1}\left(y \mid I_{C}\right)=\operatorname{Tr}\left(y ; 0, \bar{y}_{\mathrm{S}}^{*}\right)$ for $m_{1}^{Y} \in\left[0, \bar{y}_{\mathrm{S}}^{*}\right]$ and $f_{1}\left(x_{1,1} \mid I_{C}\right)=\operatorname{Tr}\left(y ; \bar{y}_{\mathrm{S}}^{*}, 1\right)$ for $m_{1}^{Y} \in\left(\bar{y}_{\mathrm{S}}^{*}, 1\right]$ if initial technologies are the same;

(iii) in case of locally determined reputations, the partition $\mathbf{a}^{*}$ and the collusion strategy $\bar{y}_{\mathrm{S}}^{*}=\bar{y}_{\mathrm{S}}^{\text {lo* }}$ satisfy

$$
\begin{aligned}
& \lambda\left[\hat{\pi}\left(Y, Y ; \bar{y}_{\mathrm{S}}^{\mathrm{lo} *}, \mathbf{a}^{*}\right)-\hat{\pi}\left(Y, Z ; \bar{y}_{\mathrm{S}}^{\mathrm{lo}}, \mathbf{a}^{*}\right)\right]=E\left[Z \mid a_{r-1}^{*} \leq z \leq a_{r+1}^{*}\right]-a_{r}^{*} \\
& \lambda\left[\hat{\pi}\left(Y, Y ; \bar{y}_{\mathrm{S}}^{\mathrm{lo} *}, \mathbf{a}^{*}\right)-\hat{\pi}\left(Y, Z ; \bar{y}_{\mathrm{S}}^{\mathrm{lo} *}, \mathbf{a}^{*}\right)\right]=E\left[Z \mid s^{Y}, s^{Y}, \bar{y}_{\mathrm{S}}^{\mathrm{lo} *}\right]-\bar{y}_{\mathrm{S}}^{\mathrm{lo} *}
\end{aligned}
$$

\footnotetext{
${ }^{33}$ Note that we assume that the planner tosses a coin in case of $X_{2,1}=Z$ and $E\left[Y \mid I_{C}^{\mathrm{cl}}\right]=E\left[Z \mid I_{C}^{\mathrm{cl}}\right]$. This ensures harmonisation - sites adopt the same technology in $t=2$. In a companion paper we analyse the case where both sites can continue with their initial technologies. This has interesting consequences for the nature and quality of communication.
} 
for $r=1, \ldots, N^{*}-1$. For $\lambda<\bar{\lambda}_{\mathrm{cl}}^{\mathrm{lo}}, N^{*} \geq 2$ and $\bar{y}_{\mathrm{S}}^{\mathrm{lo} *}>0$, whereas for $\lambda \geq \bar{\lambda}_{\mathrm{cl}}^{\mathrm{lo}}, N^{*}=1$ and $\bar{y}_{\mathrm{S}}^{\mathrm{lo} *}=0$. That is, the agents do not send influential information on $y$ and $z$ for $\lambda \geq \bar{\lambda}_{\mathrm{cl}}^{\mathrm{lo}}$.

(iv) in case of globally determined reputations, the partition $\mathbf{a}^{*}$ satisfies

$$
\lambda\left[\hat{\pi}_{1}\left(Y, Z, Y, Y ; \mathbf{a}^{*}\right)-\hat{\pi}_{1}\left(Y, Z, Z, Z ; \mathbf{a}^{*}\right)\right]=E\left[Z \mid a_{r-1}^{*} \leq z \leq a_{r+1}^{*}\right]-a_{r}^{*}
$$

for $r=1, \ldots, N^{*}-1$. The collusion strategy $\bar{y}_{\mathrm{S}}^{*}=\bar{y}_{\mathrm{S}}^{\mathrm{gl} *}$ satisfies

$$
\lambda\left[\hat{\pi}\left(Y, Y, Y, Y ; \bar{y}_{\mathrm{S}}^{\mathrm{gl}}\right)-\hat{\pi}\left(Y, Y, Z, Z ; \bar{y}_{\mathrm{S}}^{\mathrm{gl} *}\right)\right]=E\left[Z \mid s^{Y}, s^{Y}, \bar{y}_{\mathrm{S}}^{\mathrm{gl}}\right]-\bar{y}_{\mathrm{S}}^{\mathbf{g l}} .
$$

Moreover, for any finite $\lambda, N^{*} \geq 2$. For $\lambda<\bar{\lambda}_{\mathrm{cl}}^{\mathrm{gl}} \bar{y}_{\mathrm{S}}^{\mathrm{gl} *}>0$, whereas for $\lambda \geq \bar{\lambda}_{\mathrm{cl}}^{\mathrm{gl}} \bar{y}_{\mathrm{S}}^{\mathrm{gl} *}=0$. That is, in case agents initially used different strategies, agents send influential information about $y$ and $z$ for any finite $\lambda$. If agents initially used the same technology, they do not send influential information about the technology's values for $\lambda \geq \bar{\lambda}_{\mathrm{cl}}^{\mathrm{gl}}$.

In case agents used different technologies, the communication strategy is a partition strategy. Eqs (9) and (11) determine the partitioning in case of local and global markets, respectively. If agent 1 observes a value $y$ he has to decide how to rank his technology. The higher the rank is, the more likely it becomes that the center chooses his technology. This suggests that his technology is the better one. As a result, agent 1 enjoys a reputational benefit. Ranking it highly also has a cost. If $z>y$ but agent 2 does not rank $Z$ as highly as 1 ranks $Y$, the center forces both agents to choose $Y$, the inferior technology in period 2 . This possibility stops the agent from ranking his technology too highly. The left-hand sides of the equations state the net reputational value of continuing with one's technology. For $y=a_{r}^{*}$, this gain is exactly offset by a loss in expected project value due to continuation: the agent is indifferent between using two adjacent ranks (messages) to describe the value of technology $Y$. Sending one message rather than the other changes the choice of the center only for $z \in\left(a_{r-1}^{*}, a_{r+1}^{*}\right)$, see the right-hand side of (9) and (11).

In terms of informativeness, a partition strategy is in between the truthful revelation that characterizes communication in case of a decentralized with locally determined reputations and the absence of communication in case of decentralized process and globally determined reputations. That is, as a result of the move from decentralized learning to centralized learning communication deteriorates in the former case but improves in the latter. In the former 
case, the loss of an agent's decision-making power and its uploading to the center means that an agent starts to use his communication to indirectly influence the perception of his ability. The quality of communication drops. In case of globally determined reputations, the loss of decision-making power makes that an agent becomes cautious when communicating: given the communication strategy of the other agent, his own exaggerated claims are no longer costless but can lead to an inferior choice at the agent's own site.

To explain why influential communication among agents and centre remains possible for any finite $\lambda$ in case reputations can be based on comparisons across sites but vanishes for $\lambda \geq \bar{\lambda}_{\mathrm{cl}}^{\text {lo }}$ in case of locally determined reputations, it is useful to start by comparing the present model with the existing literature that uses cheap talk. As noted in the related literature section, the existing literature focuses on situations in which the difference in preferences between Sender ( $S$, here agent) and Receiver ( $R$, here center) is exogenously given. Consider the leading example introduced by Crawford and Sobel, the uniform quadratic case, in which $U_{S}(d, y)=-(d-(y+b))^{2}$ and $U_{R}(d, y)=-(d-y)^{2}$ with $y \in[0,1]$ being the state variable that is known to $S$ only, $d \in[0,1]$ the decision that is taken by $R$. The parameter $b>0$ captures the difference in preference between $S$ and $R$. Its exogenously specified value determines the maximum number of intervals (ranks) in the communication strategy of $S$, and, for a given $N$, the vector a $(N)$. In our model, the difference in preference equals $\lambda \hat{\pi}(\cdot)$, where $\hat{\pi}(\cdot)$ is determined in equilibrium. This endogeneity may make that agents send relevant information about the state for any finite $\lambda$. Indeed, proposition 4, part iv states that agents send relevant information about the technology's values for any finite $\lambda$ in case they started out with different technologies and markets are global.

This difference is illustrated in Figure 4. Panel a shows the determination of the equilibrium value $a_{1}^{*}$ in the uniform-quadratic case of Crawford and Sobel. For $N=2$, the value of $a_{1}^{*}$ solves $a_{1}=\frac{1}{2}-2 b$, see e.g. Gibbons (1992, p. 216). This equality can also be written as

$$
b=\frac{1}{2}\left(\frac{1}{2}-a_{1}\right) \text {. }
$$

The LHS captures the difference in preference alignment. It determines the equilibrium value $a_{1}^{*}$. The LHS (RHS) of (13) is plotted as a dotted (drawn) line in panel a. For the Sender to send relevant information $b<1 / 4$ must hold. 
Figure 4: Determination of the partition in the communication strategy. Communication limited to at most two ranks. Panel (a) shows the canonical uniform-quadratic case of Crawford and Sobel. Panel (b) shows the case of centralized learning from others and globally determined reputations.

(a) Crawford and Sobel

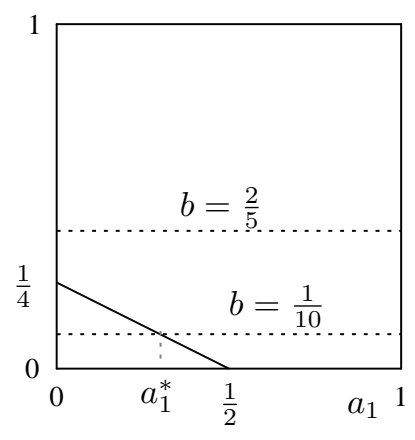

(b) Centralization / Global

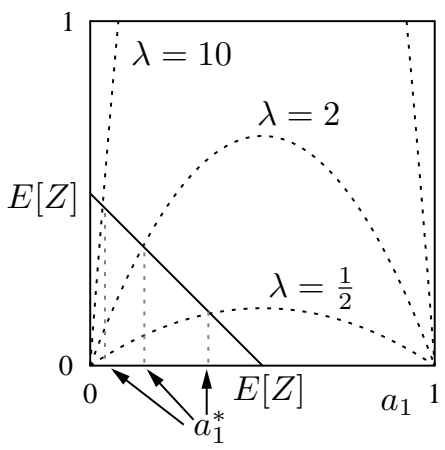

Panel $\mathrm{b}$ shows the determination of the equilibrium value $a_{1}^{*}$ in case communication between the agents and center is limited to at most two ranks and reputations are determined globally. With at most two ranks, (11) reduces to ${ }^{34}$

$$
\lambda \frac{4 \pi}{1+\pi} F\left(a_{1}\right)\left(1-F\left(a_{1}\right)\right)=E[Z]-a_{1} .
$$

The dotted lines represent the LHS for various values of $\lambda$. The reputational gap, the source of the difference in preference alignment, depends on the equilibrium value $a_{1}^{*}$ and equals zero for $a_{1}=0$. The drawn line graphs the RHS. The graphs illustrates that for any finite $\lambda$, there is a unique $a_{1}^{*}>0$. That is, for any finite weight $\lambda$ that the agent puts on his reputation, the agent uses (at least) two ranks.

The key to understand why communication among agents and centre remains possible for any finite $\lambda$ is the fact that the reputational gap equals zero for $a_{1}=0$. If agents use different technologies and $a_{1}=0$, the center decides on the technology that is to be used in $t=2$ by tossing a coin. With globally determined reputations, it is known that the initial distribution of technologies equaled $Y, Z$. As a result, the decision of the center does not add any information on the relative values of the technologies nor on the ability of the agents. Hence, $\hat{\pi}_{1}\left(Y, Z, Y, Y ; a_{1}=0\right)=\hat{\pi}_{1}\left(Y, Z, Z, Z ; a_{1}=0\right)$. Instead, with locally

\footnotetext{
${ }^{34}$ See the proof of Proposition 4.
} 
determined reputations, relevant communication about the value of a technology is not possible for $\lambda \geq \bar{\lambda}_{\mathrm{cl}}^{\mathrm{lo}}$ as even for $a_{1}=0$ (i.e., one rank only) and $\bar{y}_{\mathrm{S}}^{\text {lo* }}=0$ the reputational gap does not vanish but equals $\frac{4 \pi^{2}}{\left(3+\pi^{2}\right)(1+\pi)} \cdot 35$ The reason is that it is not known whether agents initially used the same technologies or different ones. If an agent is forced to change technology, it is inferred that agents must initially have used different technologies and that next the center tossed a coin. The deduced difference in initial technology hurts an agent's reputation. If instead an agent must continue his initial technology this may also mean that both agents initially used the same technology. The latter makes it more likely that the agents received a correct signal. As a result, continuation boosts an agent's reputation, and the reputational gap continues to exist even for $a_{1}=0$.

\section{Welfare Comparisons}

What are the consequences of the assignment of decision rights? Does it depend on the information on which reputations are based? We consider for each process the expected value of the technology that is in use at site 1 in period 2, assuming that 1 starts with $Y$, $E\left[X_{1,2} \mid s^{Y}, \lambda, \pi\right]$. The expectation is taken over $y$, and before 1 knows agent 2's technology in period 1, assuming of course equilibrium behaviour. The theoretical maximum value is $E[Y \mid y>z]$, which obtains if agent 1 chooses the better technology in period 2 with probability one. No process generates this value, unless $\pi=1$ in which case the better technology is identified in $t=1$. Absent perverse behaviour, the theoretical minimum value is $\pi E[Y \mid y>z]+(1-\pi) E[Y]$. This is the expected value in case the technology adopted at site 1 in $t=2$ equals the first period choice with probability one, independent of the experience gained with the technologies in $t=1$ throughout the economy.

To focus on differences in value creation thanks to learning from own past behaviour and from the experience of others, we transform $E\left[X_{1,2} \mid s^{Y}, \lambda, \pi\right]$ using the following formula,

$$
W(\lambda, \pi)=\frac{E\left[X_{1,2} \mid s^{Y}, \lambda, \pi\right]-(\pi E[Y \mid y>z]+(1-\pi) E[Y])}{E[Y \mid y>z]-(\pi E[Y \mid y>z]+(1-\pi) E[Y])} * 100 \%
$$

That is, $W(\lambda, \pi) \in[0 \%, 100 \%]$ captures value creation thanks to learning, over and above

\footnotetext{
${ }^{35}$ For the derivation, see the proof of Proposition 4.
} 
the minimum value, as a percentage of what is maximally attainable. We refer to it as 'welfare.'

\subsection{Decentralized learning: welfare comparisons}

In this subsection, we compare with each other isolated agents, decentralized learning with locally determined reputations, and decentralized learning with globally determined reputations. Key to welfare comparisons are (i) the information agents have, and (ii) the degree to which they use it in the various situations. Consider (i). By definition, an isolated agent only knows the value of his own technology, and does not know what technology has been adopted at the other site. We know from Propositions 2 and 3 that in case of decentralized learning and globally determined reputations for any $\lambda>0$ agent 1 also knows $X_{2,1}$ (but not $x_{2,1}$ if $X_{2,1}=Z$ ), and that if reputations are locally determined he knows both $X_{2,1}$ and $x_{2,1}$. If an agent does not care about his reputation, additional information can only lead to an increase in welfare. This implies that there is some $\lambda_{1}>0$ such that for all $\lambda \in\left(0, \lambda_{1}\right)$ additional information is also welfare-enhancing: $W_{\mathrm{ia}}(\lambda, \pi)<W_{\mathrm{dl}}^{\mathrm{gl}}(\lambda, \pi)<W_{\mathrm{dl}}^{\mathrm{lo}}(\lambda, \pi){ }^{36}$

Consider (ii). Propositions 1-3 show that the degree to which information is used depends on the strength of reputational concerns. In particular, they establish for the three cases the values of $\lambda$ above which an agent ignores all information and simply continues with his initial choice of technology. These values are $\bar{\lambda}_{\mathrm{ia}}=E[Z] / \pi, \bar{\lambda}_{\mathrm{dl}}^{\mathrm{lo}}=1 / \pi$, and $\bar{\lambda}_{\mathrm{dl}}^{\mathrm{gl}}=$ $E[Z](1+\pi) / \pi$ for an isolated agent, an agent under a decentralized process and locally determined reputations, and an agent under a decentralized process and globally determined reputations, respectively. As $\bar{\lambda}_{\mathrm{ia}}<\bar{\lambda}_{\mathrm{dl}}^{\mathrm{lo}}, \bar{\lambda}_{\mathrm{dl}}^{\mathrm{gl}}$, an isolated agent stops using information for a lower value of $\lambda$ than a decentralized agent. ${ }^{37}$ Furthermore, $\bar{\lambda}_{\mathrm{dl}}^{\mathrm{gl}}<\bar{\lambda}_{\mathrm{dl}}^{\text {lo }}$ if and only if $E[Z](1+\pi)<1$. If this inequality holds, the ordering for sufficiently high values of $\lambda$, $\lambda>\lambda_{2}$ for some $\lambda_{2}>0,{ }^{38}$ is the same as for low values of $\lambda$ : a decentralized process and

\footnotetext{
${ }^{36}$ Recall that ia stands for isolated agents, $\mathrm{dl}(\mathrm{cl})$ for decentralized (centralized ) learning, and gl (lo) for globally (locally) determined reputations.

${ }^{37}$ Note that the 1 in $\bar{\lambda}_{\mathrm{dl}}^{\text {lo }}=1 / \pi$ is the upperbound of the support of $f_{X}$. The inequality therefore holds independent of the chosen support.

${ }^{38}$ By continuity, $\lambda_{2}<\bar{\lambda}_{\mathrm{dl}}^{\mathrm{gl}}$.
} 
locally determined reputations leads to the highest project value, next comes decentralized learning with globally determined reputations, and isolated agents perform the worst.

To understand the condition $E[Z](1+\pi)<1$, it is important to realize that information has two roles. On the one hand, additional information helps the agent in identifying the better technology. In case of locally determined reputations, agent 1 knows the value of the other technology. The difference $z-y$ can be as large as 1 . In case of globally determined reputations, agent 1 does not know the value of the other technology. Instead, he can only calculate $E[Z]-y$. This difference is at most $E[Z]$. Hence, ceteris paribus, for all information about $Z$ to be ignored and for the agent to continue with $Y, \lambda$ should be larger when his reputation is locally determined than when it is globally determined. On the other hand, additional information helps the market in evaluating an agent's ability. If a market cannot compare across sites, then reputation-wise more is at stake when the agent takes a decision relative to the case where a market can compare across sites. In the latter case the market already knows whether agents used the same or different technologies. If agent 1 were to continue with $Y$, rather than to switch to $Z$, independent of what he knows about $Z$, then the reputational gap equals $\pi$ if markets have access to local information only and $\pi /(1+\pi)<\pi$ if markets can compare across sites. Hence, ceteris paribus, $\lambda$ should be larger in the latter case than in the former case for information about $Z$ to be ignored and for the agent to continue with $Y$. The inequality $E[Z](1+\pi)<1$ holds if it is sufficiently hard to identify the better technology ( $\pi$ low), and if the unconditional expected value $E[Z]$ of a technology is sufficiently low. In case of the uniform distribution or any other symmetric distribution it holds.

In Figure 5, we compare value, as measured by $W$, for decentralized learning with reputations that are locally and globally determined and for isolated agents under the assumption that the value of technology $X \in\{Y, Z\}$ is uniformly distributed, $f_{X}(x)=1$ on $[0,1]$, and that $\pi=\frac{1}{2} \cdot 39$

Figure 5 illustrates a number of points. First, learning from one's own past behaviour and from others potentially boosts welfare enormously. For $\lambda$ close to zero, an isolated agent who is of high ability with probability $\pi=1 / 2$ and learns from his own experience only

\footnotetext{
${ }^{39}$ For $f_{X}=1$ and $\pi=1 / 2, E[Y \mid y>z]=2 / 3$ and $\pi E[Y \mid y>z]+(1-\pi) E[Y]=7 / 12$.
} 
Figure 5: $W(\lambda, \pi)$ for isolated agents and for decentralized learning from others with locally and globally determined reputations. $f_{X}=1$ and $\pi=1 / 2$ such that $\bar{\lambda}_{\mathrm{ia}}=1, \bar{\lambda}_{\mathrm{dl}}^{\mathrm{gl}}=3 / 2$, and $\bar{\lambda}_{\mathrm{dl}}^{\mathrm{lo}}=2$.

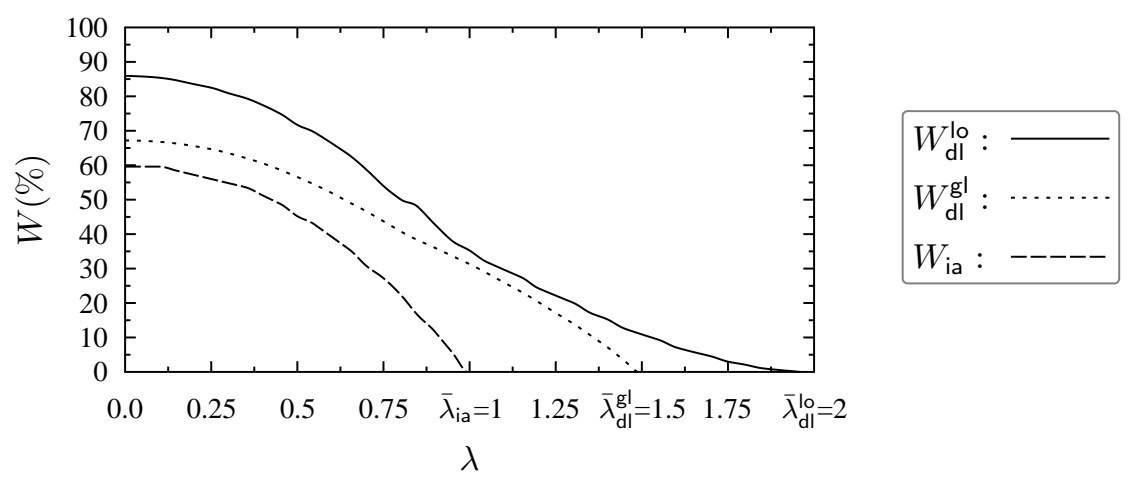

can capture $60 \%$ of the increase in expected project value. learning further increases this percentage. Second, if markets can compare agents' behaviour across sites, this reduces the positive effect of learning from others. The main reason is that communication breaks down when markets can make comparisons. Third, the relative performance does not change in $\lambda$. Additional calculations (not reported here) show that this is true independent of the value of $\pi$. The following Proposition sums up. ${ }^{40}$

Proposition 5 For any $f_{X}$ and $\pi$, there exists a $\lambda_{1}>0$ such that $W_{\mathrm{ia}}(\lambda, \pi)<W_{\mathrm{dl}}^{\mathrm{gl}}(\lambda, \pi)<$ $W_{\mathrm{dl}}^{\mathrm{lo}}(\lambda, \pi)$ for all $\lambda<\lambda_{1}$. Furthermore, for any $f_{X}$ and $\pi$ such that $E[Z](1+\pi)<1$, there exists a $\lambda_{2}>0$ such that $W_{\mathrm{ia}}(\lambda, \pi)<W_{\mathrm{dl}}^{\mathrm{gl}}(\lambda, \pi)<W_{\mathrm{dl}}^{\mathrm{lo}}(\lambda, \pi)$ for all $\lambda>\lambda_{2}$. If instead $f_{X}$ and $\pi$ satisfy $1<E[Z](1+\pi)$, then there exists a $\lambda_{3}>0$ such that $W_{\mathrm{ia}}(\lambda, \pi)<W_{\mathrm{dl}}^{\text {lo }}(\lambda, \pi)<$ $W_{\mathrm{dl}}^{\mathrm{gl}}(\lambda, \pi)$ for $\lambda>\lambda_{3}$. For $f_{X}=1$, the uniform distribution, $W_{\mathrm{ia}}(\lambda, \pi)<W_{\mathrm{dl}}^{\mathrm{gl}}(\lambda, \pi)<$ $W_{\mathrm{dl}}^{\mathrm{lo}}(\lambda, \pi)$ holds for all $\lambda$ and $\pi$.

\subsection{Centralized learning: welfare comparisons}

In this subsection, we compare with each other isolated agents, centralized learning with locally determined reputations, and centralized learning with globally determined reputa-

${ }^{40}$ If $\lambda \in\left(\bar{\lambda}_{\mathrm{dl}}^{\mathrm{gl}}, \bar{\lambda}_{\mathrm{dl}}^{\mathrm{lo}}\right)$, then $W_{\mathrm{ia}}(\lambda, \pi)=W_{\mathrm{dl}}^{\mathrm{gl}}(\lambda, \pi)=0<W_{\mathrm{dl}}^{\mathrm{lo}}(\lambda, \pi)$. If $\lambda \geq \bar{\lambda}_{\mathrm{dl}}^{\mathrm{lo}}$, then, $W_{\mathrm{ia}}(\lambda, \pi)=W_{\mathrm{dl}}^{\mathrm{gl}}(\lambda, \pi)=$ $W_{\mathrm{dl}}^{\mathrm{lo}}(\lambda, \pi)=0$. These cases are ignored in Proposition 5 . 
tions. Propositions 1 and 4 allow us to compare welfare $W$ in case of centralized learning and isolated agents. ${ }^{41}$

Proposition 6 For any $f_{X}$ and $\pi$, there exists a $\lambda_{4}>0$ such that $W_{\mathrm{ia}}(\lambda, \pi)<W_{\mathrm{cl}}^{\mathrm{lo}}(\lambda, \pi), W_{\mathrm{cl}}^{\mathrm{gl}}(\lambda, \pi)$ for all $\lambda \in\left(0, \lambda_{4}\right)$. Furthermore, for any $f_{X}$ and $\pi$ there exists a $\lambda_{5}>0$ such that $W_{\mathrm{ia}}(\lambda, \pi)<W_{\mathrm{cl}}^{\mathrm{lo}}(\lambda, \pi)<W_{\mathrm{cl}}^{\mathrm{gl}}(\lambda, \pi)$ for all $\lambda>\lambda_{5}$. In addition, for $f_{X}=1$, the uniform distribution, $W_{\mathrm{ia}}(\lambda, \pi)<W_{\mathrm{cl}}^{\mathrm{gl}}(\lambda, \pi), W_{\mathrm{cl}}^{\mathrm{lo}}(\lambda, \pi)$ holds for all $\lambda>0$ and $\pi$.

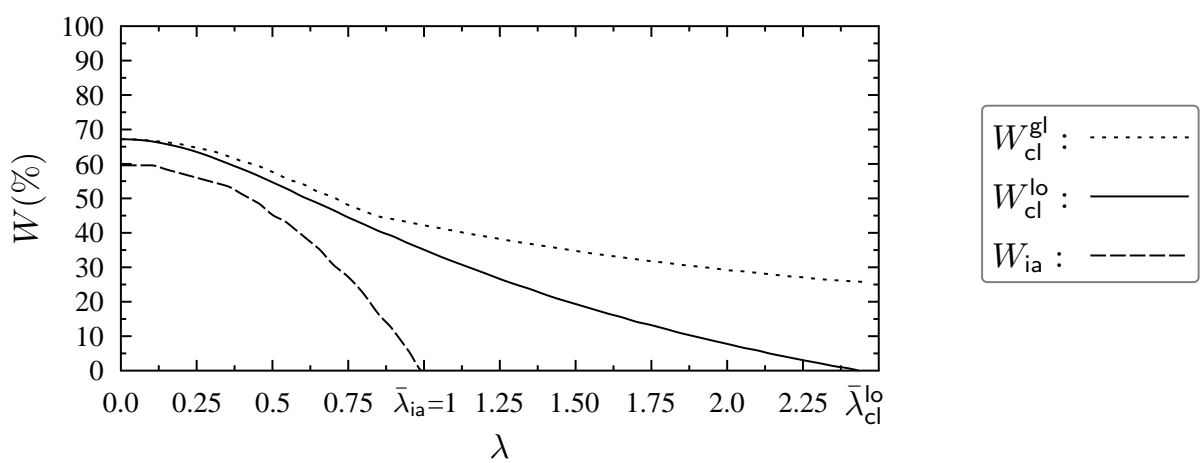

Figure 6: $W(\lambda, \pi)$ for isolated agents and for centralized learning from others with locally and globally determined reputations. $W(\lambda, \pi)$ in case of centralized learning is based on a partition strategy with at most two ranks. $f_{X}=1$ and $\pi=1 / 2$, such that $\bar{\lambda}_{\text {ia }}=1, \bar{\lambda}_{\mathrm{cl}}^{\text {lo }}=2 \frac{7}{16}$.

Proposition 6 is illustrated in Figure 6 for the uniform distribution and $\pi=1 / 2$. We have imposed that communication with the center is limited to at most two ranks in case agents initially used different technologies. Clearly, if agents can learn from others welfare improves. Because of our limitation to at most two ranks, the graph understates the benefits for low values of $\lambda$. In fact, for $\lambda=0$, agents would truthfully reveal their private information and the performance of a centralized learning process would equal that of a decentralized learning process. We then know from Figure 5 that $W \approx 86 \%$ rather than $W \approx 68 \%$ as shown in the graph. Note that when markets can compare technologies across sites, the positive effect of learning further increases, especially for high values of $\lambda$. This stems from the fact that communication between agents and center remains influential for any finite $\lambda$

\footnotetext{
${ }^{41}$ If $\lambda \geq \bar{\lambda}_{\mathrm{cl}}^{\mathrm{lo}}$, then, $W_{\mathrm{ia}}(\lambda, \pi)=W_{\mathrm{cl}}^{\mathrm{lo}}(\lambda, \pi)=0$. This case is ignored in Proposition 6 .
} 
in case of globally determined reputations, whereas it dies out for high values of $\lambda$ in case of locally determined reputations.

\subsection{Further comparisons}

In the previous two subsections we have analysed how welfare changes if, for a given assignment of decision rights, the information on which the perceptions of agents' abilities are based changes from local to global. In this subsection we turn to the complementary question, and analyse, for a given information base on which reputations can be based, the conditions that determine whether a decentralized process or a centralized process performs best.

If reputations are locally determined, the learning process that is best depends fundamentally on the parameters of the model. ${ }^{42}$

\section{Proposition 7 Suppose}

$$
\frac{1}{E[Z]}>\frac{\left(3+\pi^{2}\right)(1+\pi)}{4 \pi} .
$$

Then, in case of locally determined reputations, there exists a $\lambda_{6}<\bar{\lambda}_{\mathrm{cl}}^{\text {lo }}$ such that welfare $W(\lambda, \pi)$ is higher with decentralized than with centralized learning for all $\lambda>\lambda_{6}$.

If condition (16) is met, there are values of $\lambda$ such that under a decentralized process the technology adoption decision in $t=2$ depends on the observed values $y$ and $z$, whereas in a centralized process, agents do not transmit useful information about their technologies. As a result, expected welfare is higher in case of decentralized learning.

Note that $\left(3+\pi^{2}\right)(1+\pi) / 4 \pi>2$ for all $\pi \cdot 1 / E[Z]$ is the ratio of the upperbound of the support and the expected value of the technology. Hence, the ratio should exceed 2 for there to be values of $\pi$ such that a decentralized process outperforms a centralized one for high values of $\lambda$. The uniform distribution cannot meet this condition. Does this mean that welfare is higher under a centralized process than under a decentralized process in case of the uniform distribution for all $\lambda$ and $\pi$ ? The next proposition provides sufficient conditions on $\lambda$ and $\pi$ such that a decentralized process outperforms a centralized process.

\footnotetext{
${ }^{42}$ If $\lambda \geq \bar{\lambda}_{\mathrm{cl}}^{\mathrm{lo}}$, then $W_{\mathrm{dl}}^{\mathrm{lo}}(\lambda, \pi)=W_{\mathrm{cl}}^{\mathrm{lo}}(\lambda, \pi)$. This case is ignored in Proposition 7 .
} 
Proposition 8 Assume reputations are locally determined and technology values are uniformly distributed. Then, for any $\pi$ there are values of $\lambda, \lambda \in[\underline{\lambda}(\pi), \bar{\lambda}(\pi)]$, with $0<$ $\underline{\lambda}(\pi)<\bar{\lambda}(\pi)$, such that welfare $W(\lambda, \pi)$ is higher under decentralized than under centralized learning.

What learning process is best if reputations can also be based on comparisons across sites?

Proposition 9 In case of globally determined reputations, and for any $f_{X}, \pi$, and $\lambda$, welfare $W(\lambda, \pi)$ is higher with centralized than with decentralized learning.

The main benefit of moving from a decentralized process to a centralized one in case reputations can also be based on comparisons across sites is the restoration of communication when agents initially used different technologies. The proof establishes that even if agents in a centralized learning process were to limit themselves to a communication strategy consisting of at most two ranks - and choose $a_{1}^{*}$ optimally - welfare goes up. This suggests that the welfare difference can be substantial for low values of $\lambda$, as such values allow for richer communication (i.e., finer partitions).

\section{Concluding Remarks}

An important objective of this paper was to gain insight into the effects of alternative learning processes on the quality of decisions in situations where information is dispersed among agents, and agents are concerned about their reputations. Our analysis focuses on two broad features of decision-making processes: the extent of a centralized process and whether reputations of decision-makers are based on local information only, or can also be based on comparisons across sites. We believe that our focus enabled us to derive a couple of interesting results. By focusing on these two broad features, we have abstracted from other features of learning processes. Here we would like to elaborate on some of the specific assumptions we have made.

Centralization. One important assumption is that in a centralized process the center always acts in the general interest. In reality, there is little reason to put so much confidence in central bodies. For example, a center may be biased towards one of the technologies 
because of favoritism. Alternatively, a center may be biased because somehow its name is connected to one of the technologies. Of course, our assumption of a "benevolent" center provides too favourable a picture of centralized processes.

Information. We have described the private information that agents have as non-verifiable, and communication as cheap talk. Although this may well reflect an important part of information agents have gained locally, they may also have verifiable information. Such information can be checked by other agents. If it is unknown whether an agent actually possesses information that is decision-relevant to another agent, the former may have an incentive to selectively withhold his private information from the latter, see e.g. Milgrom and Roberts (1986). How does the presence of verifiable information change our findings? Although the nature of information manipulation changes, the incentives to manipulate continue to be determined by the interplay of the decision rights and the information on which reputations are based. As a result, the quality of information exchange depends in essentially the same way on these same two factors. Consider decentralized decision-making with locally determined reputations. The fact that an agent's reputation is independent of what the other agent does and that an agent can decide himself what technology he uses next makes that revealing all positive and negative pieces of information is a weakly dominant strategy. If reputations reputations are also based on comparisons across sites (and decentralised learning), it is important from a reputational perspective to convince the other agent to switch to "your" technology. As a result, any negative information will be withheld. The introduction of centralised decision-making in such a situation gives rise to the selective revelation of negative information. On the one hand, as the agent at a site loses decision-making power, he wants to make sure that the center is well-informed. On the other hand, his reputational concerns imply that he wants the center to impose "his" technology at either site. Ceteris paribus, the more damaging negative information is for the technological value, the more likely it is that the information is revealed. Similarly, the more damaging negative information is for his reputation, the less likely it becomes that this information is revealed.

In our model, signals are for free. However, one can easily imagine situations where agents can increase the probability of receiving an informative signal by putting more effort 
in investigating technologies. We consider modeling agents' effort decisions as a promising extension of our model. We expect that reputational concerns do not only lead to distortions in communication and decisions, but that they may also induce agents to put more effort in investigating technologies, see e.g. Suurmond et al. (2004).

Decision rights. We have limited attention to a centralized process and a decentralized process. A possible third organizational structure is a committee consisting of the two agents that makes a collective decision in period 2 on the basis of some voting rule. Visser and Swank (2007) analyze communication and voting in committees in the presence of reputational concerns.

Our approach is particularly relevant for situations where agents independently gained experiences that are worth sharing. In our model, period 1 represents history. However, in other situations experience still has to be gained. Then, some planner could opt for ignoring signals and assign one technology to agent 1 and the other technology to agent 2 . Such a procedure is likely to weaken reputational concerns as the technology decisions are no longer linked to signals. Moreover, it allows for learning in period 2. It is easy to show that assigning technologies in period 1 is optimal if signals are not very informative. The first-period costs of ignoring signals are then small.

\section{Appendix}

Proof of lemma 1: Consider (1) in the text. (a) As $\operatorname{Pr}\left(\bar{\theta} \mid s^{Y}, 0\right)=0, E\left[Z \mid s^{Y}, 0\right]=E[Z]$. Similarly, as $\operatorname{Pr}\left(\bar{\theta} \mid s^{Y}, 1\right)=1$, then $E\left[Z \mid s^{Y}, 1, \bar{\theta}\right]=E[Z]$, and therefore $E\left[Z \mid s^{Y}, 1\right]=E[Z]$.

Moreover, $E\left[Z \mid s^{Y}, y, \bar{\theta}\right]<E[Z]$ for $y \in(0,1)$, as the term on the LHS is the expected value of the truncated distribution on $[0, y)$. (b) To determine the derivative, use Bayes' rule to write $\operatorname{Pr}\left(\bar{\theta} \mid s^{Y}, y\right)=2 F(y) \pi /(2 F(y) \pi+(1-\pi))$. Also, $E\left[Z \mid s^{Y}, y, \bar{\theta}\right]=\int_{0}^{y} t f(t) d t / F(y)$. One can verify that $\partial \operatorname{Pr}\left(\bar{\theta} \mid s^{Y}, y\right) / \partial y=\operatorname{Pr}\left(\bar{\theta} \mid s^{Y}, y\right)\left(1-\operatorname{Pr}\left(\bar{\theta} \mid s^{Y}, y\right)\right) \frac{f(y)}{F(y)}>0$, and that $\partial E\left[Z \mid s^{Y}, y, \bar{\theta}\right] / \partial y=\left(y-E\left[Z \mid s^{Y}, y, \bar{\theta}\right]\right) \frac{f(y)}{F(y)}$. Hence,

$$
\partial E\left[Z \mid s^{Y}, y\right] / \partial y=\operatorname{Pr}\left(\bar{\theta} \mid s^{Y}, y\right) \frac{f(y)}{F(y)}\left(y-E\left[Z \mid s^{Y}, y\right]\right),
$$


from which it follows immediately that $E\left[Z \mid s^{Y}, y\right]$ is decreasing for $y<E\left[Z \mid s^{Y}, y\right]$ and increasing for $y>E\left[Z \mid s^{Y}, y\right]$. Hence, $y=E\left[Z \mid s^{Y}, y\right]$ has a unique solution.

Proof of Proposition 1: First, $\hat{\pi}(Y Y ; \bar{t})=\operatorname{Pr}(\bar{\theta} \mid Y Y ; \bar{t})$ in $(2)$. Use $\operatorname{Pr}(Y Y \mid \bar{\theta})=$ $\operatorname{Pr}(y \geq \bar{t} \mid \bar{\theta}) / 2=\left(1-F(\bar{t})^{2}\right) / 2$ and $\operatorname{Pr}(Y Y \mid \underline{\theta})=\operatorname{Pr}(y \geq \bar{t} \mid \underline{\theta}) / 2=(1-F(\bar{t})) / 2$, and apply Bayes rule (analogously for $\hat{\pi}(Y Z ; \bar{t})$. Clearly, for given reputations the equilibrium strategy is a single threshold strategy with $\bar{y}_{\mathrm{ia}}^{*}$ satisfying (3). Given this strategy, equilibrium reputations are as in (2) with $\bar{t}=\bar{y}_{\mathrm{ia}}^{*} \leq \bar{y}_{\mathrm{ia}}^{F B}$. To see that $\bar{y}_{\mathrm{ia}}^{*}$ is a decreasing function of $\lambda$ for $\lambda \leq \bar{\lambda}_{\text {ia }}$, define $\delta:=\bar{y}_{\mathrm{ia}}^{F B}-\bar{y}_{\mathrm{ia}}$ and $\Delta \hat{\pi}:=\hat{\pi}(Y Y)-\hat{\pi}(Y Z)$. Then $(\delta, \Delta \hat{\pi}) \in L:=[0, E[Z]] \times[0,1]$, and so $L$ is a complete lattice. It follows from Lemma 1 that (3) can be written as $\delta=f_{1}(\Delta \hat{\pi}, \lambda)$. It follows from (3) that the function $f_{1}$ satisfies $\partial f_{1} / \partial \Delta \hat{\pi}, \partial f_{1} / \partial \lambda>0$, and from (2) that $\Delta \hat{\pi}=f_{2}(\delta)$ is an increasing function of $\delta$. Hence, we can apply Theorem 3 in Milgrom and Roberts (1994). The set of fixed points of $f: L \times \mathbb{R}^{+} \rightarrow L$ is non-empty and equals the set of equilibria, and $\delta^{*}=\bar{y}_{\mathrm{ia}}^{F B}-\bar{y}_{\mathrm{ia}}^{*}$ is increasing in $\lambda$. Moreover, in case this set is not a singleton, both the highest and the lowest fixed point are increasing in $\lambda$. It is straightforward to check that for $\lambda \geq \bar{\lambda}_{\mathrm{ia}}, \bar{y}_{\mathrm{ia}}^{*}=0$.

Proof of Proposition 2: The equilibrium belief functions follow immediately from the equilibrium message strategies. That the decision strategy is a double-threshold strategy follows from the analysis preceding the statement of the proposition. Finally, note that for $\bar{t}_{\mathrm{S}}^{*}=0$, the RHS of (4) equals $E[Z]$, and therefore $\bar{t}_{\mathrm{D}}^{*}=E[Z]$, and thus $\lambda=\underline{\lambda}_{\mathrm{dl}}^{\text {lo }}$. Finally, if $\bar{t}_{\mathrm{S}}^{*}=0$ and $\bar{t}_{\mathrm{D}}^{*}=1, \hat{\pi}(Y Y ; 0,1)=\pi$ (as agent uses pooling strategy) and $\hat{\pi}(Y Z ; 0,1)=0$ (this is an out-of-equilibrium belief, the limit of $\hat{\pi}(Y Z)$ in case $\bar{t}_{\mathrm{D}}^{*} \uparrow 1$ ) such that for $\lambda \geq \bar{\lambda}_{\mathrm{dl}}^{\text {lo }}$, the agents indeed continue with their initial technologies no matter what.

Proof of Proposition 3: First, the reputations. $\hat{\pi}_{1}(Y Y Y Y ; \bar{c})=\operatorname{Pr}(\bar{\theta} \mid Y Y Y Y ; \bar{c})$. Write $F(\bar{c})=F$. Use $\operatorname{Pr}(Y Y Y Y \mid \bar{\theta})=\operatorname{Pr}(Y Y Y Y \mid \bar{\theta}, y>z) / 2=(1+\pi) \operatorname{Pr}(y>\bar{c} \mid y>z) / 4=$ $(1+\pi)\left(1-F^{2}\right) / 4$ and $\operatorname{Pr}(Y Y Y Y \mid \underline{\theta})=(1+\pi)\left(1-F^{2}\right) / 8+(1-\pi)(1-F)^{2} / 8$, and so $\hat{\pi}(Y Y Y Y ; \bar{c})=(1+F) \frac{1+\pi}{1+\pi^{2}+2 F \pi} \pi>\pi$. Similarly, $\hat{\pi}(Y Y Z Z ; \bar{c})=F \frac{\pi+1}{1+\pi(2 F-2+\pi)} \pi$. One can check that $\Delta \hat{\pi}(\mathrm{S}):=\hat{\pi}(Y Y Y Y ; \bar{c})-\hat{\pi}(Y Y Z Z ; \bar{c})$ is decreasing in $\bar{c}$. In particular, for $\bar{c}_{\mathrm{S}}^{*}=0$, the gap equals $\frac{1+\pi}{1+\pi^{2}} \pi$. Also, $\hat{\pi}_{1}(Y Z Y Y)=\operatorname{Pr}(\bar{\theta} \mid Y Z Y Y)$. From $\{Y, Z, Y, Y\}$ the market deduces that $y>z$ in case of both first-best and equilibrium behaviour. Thus, $\operatorname{Pr}(Y Z Y Y \mid \bar{\theta})=(1-\pi) / 4\left(\right.$ as $\theta_{2}=\underline{\theta}$ for $\left.X_{2,1}=Z\right)$ and $\operatorname{Pr}(Y Z Y Y \mid \underline{\theta})=(1-\pi) / 8$, and ap- 
ply Bayes rule. Finally, $\hat{\pi}_{1}(Y Z Y Z)=\operatorname{Pr}(\bar{\theta} \mid Y Z Y Z)$. From $\{Y, Z, Y, Z\}$ the market deduces that $(y, z) \in A:=\left\{(y, z) \mid y, z<\bar{c}_{\mathrm{D}}^{*}\right.$ or $\left.y, z>\bar{c}_{\mathrm{D}}^{*}\right\}$. Use $\operatorname{Pr}(Y Z Y Z \mid \bar{\theta})=\frac{1-\pi}{2} \operatorname{Pr}(A \mid y>z) \frac{1}{2}$, $\operatorname{Pr}(Y Z Y Z \mid \underline{\theta})=\frac{1}{2} \frac{1-\pi}{2} \operatorname{Pr}(A \mid y>z) \frac{1}{2}+\frac{1}{2} \frac{1+\pi}{2} \operatorname{Pr}(A \mid z>y) \frac{1}{2}$, and $\operatorname{Pr}(A \mid z>y)=\operatorname{Pr}(A \mid y>z)$ (as $Y$ and $Z$ are iid), and apply Bayes rule. For given reputations and behaviour of 2, if $y=\bar{c}_{\mathrm{D}}^{*}$, and if 1 continues $Y$ he gets $\bar{c}_{\mathrm{D}}^{*}+\lambda \operatorname{Pr}\left(z<\bar{c}_{\mathrm{D}}^{*}\right) 2 \pi /(1+\pi)+\lambda \operatorname{Pr}\left(z \geq \bar{c}_{\mathrm{D}}^{*}\right) \pi /(1+\pi)$, whereas switching to $Z$ yields $E[Z]+\lambda \operatorname{Pr}\left(z<\bar{c}_{\mathrm{D}}^{*}\right) \pi /(1+\pi)$. Equating these expressions, one obtains (7). It is immediate that $\bar{c}_{\mathrm{D}}^{*}$ is a decreasing function of $\lambda$. The comparative statics result on $\bar{c}_{\mathrm{S}}^{*}$ uses Theorem 3 in Milgrom and Roberts (1994), see also proof of Proposition 1. The expressions for $\underline{d}_{\mathrm{dl}}^{\mathrm{gl}}$ and $\bar{\lambda}_{\mathrm{dl}}^{\mathrm{gl}}$ are then immediate.

Proof of Proposition 4: Assume $X_{1,1} \neq X_{2,1}$, that the center uses (8), that reputations are given, and that agent 2 uses the partition strategy $\left(N^{*}, \mathbf{a}^{*}\right)$ to communicate about $Z$. We show that it is then a best-reply for agent 1 to use a partition strategy with the same partitions to communicate about $Y$. We focus on the case of lo, and write $\hat{\pi}(Y, X)$ instead of $\hat{\pi}\left(Y, X ; \bar{y}_{\mathrm{S}}^{\text {lo* }}, \mathbf{a}^{*}\right)$. Derivations for the $\mathrm{gl}$ case are analogous. Let $y=a_{r}$, where we have suppressed reference to the number of partitions $N$. At this value of $y, 1$ should be indifferent between sending some $m_{r+1} \in\left[a_{r}, a_{r+1}\right)$ or some $m_{r} \in\left[a_{r-1}, a_{r}\right)$. If $z<a_{r-1}$ or $z \geq a_{r+1}$, whether 1 sends $m_{r}$ or $m_{r+1}$ does not affect the decision of the center. Hence, one can limit attention to $z \in\left[a_{r-1}, a_{r+1}\right)$. As $E\left[Z \mid s^{Y}, s^{Z}, y=a_{r}\right]=E[Z]$, $E\left[Z \mid s^{Y}, s^{Z}, y=a_{r}, \alpha \leq z \leq \beta\right]=E[Z \mid \alpha \leq z \leq \beta]$ for any pair $(\alpha, \beta)$ such that $0 \leq \alpha<$ $\beta \leq 1$. Let $p(\alpha, \beta):=F(\beta)-F(\alpha)$. Sending $m_{r+1}$ yields agent 1

$$
\begin{aligned}
& p\left(a_{r-1}, a_{r}\right)\left[a_{r}+\lambda \hat{\pi}_{1}(Y Y)\right]+\frac{1}{2} p\left(a_{r}, a_{r+1}\right)\left[a_{r}+\lambda \hat{\pi}_{1}(Y Y)\right]+ \\
& \frac{1}{2} p\left(a_{r}, a_{r+1}\right)\left[E\left[Z \mid a_{r} \leq z<a_{r+1}\right]+\lambda \hat{\pi}_{1}(Y Z)\right],
\end{aligned}
$$

whereas $m_{r}$ yields

$$
\begin{aligned}
& \frac{1}{2} p\left(a_{r-1}, a_{r}\right)\left[a_{r}+\lambda \hat{\pi}_{1}(Y Y)\right]+\frac{1}{2} p\left(a_{r-1}, a_{r}\right)\left[E\left[Z \mid a_{r-1} \leq z<a_{r}\right]+\lambda \hat{\pi}_{1}(Y Z)\right] \\
& +p\left(a_{r}, a_{r+1}\right)\left[E\left[Z \mid a_{r} \leq z<a_{r+1}\right]+\lambda \hat{\pi}_{1}(Y Z)\right] .
\end{aligned}
$$

Equating (17) and (18) shows that agent 1 is indifferent between sending $m_{r+1}$ and $m_{r}$ for $y=a_{r}$ if (9) holds.

If $X_{1,1}=X_{2,1}=Y$, it is straightforward to check that, if agent 2 uses the collusion strategy, if the center's decision strategy is as stated, and for given beliefs $\hat{\pi}$, then for agent 1 
a collusion strategy with $\bar{y}_{S}^{\text {lo* }}$ satisfying (10) is a best-reply. It is straightforward to establish that the belief function follows from applying Bayes' rule to the communication strategies of the agents, and that the center's decision strategy is a best reply given the belief function.

Consider $\bar{y}_{S}^{\text {lo* }}=0, \mathbf{a}_{1}^{*}(1)=0$ (a pooling communication strategy). To determine $\hat{\pi}(Y Y)-$ $\hat{\pi}(Y Z)$, use $\operatorname{Pr}(Y Y \mid \bar{\theta})=\operatorname{Pr}(Y Y \mid \bar{\theta}, y>z) \frac{1}{2}+\operatorname{Pr}(Y Y \mid \bar{\theta}, z>y) \frac{1}{2}=\frac{1}{8} \pi+\frac{3}{8}$, and $\operatorname{Pr}(Y Y \mid \underline{\theta})=$ $\frac{3}{8}$. Hence, $\hat{\pi}(Y Y)=\frac{3+\pi}{3+\pi^{2}} \pi$. Similarly, $\hat{\pi}(Y Z)=\frac{\pi}{\pi+1}$, such that $\hat{\pi}(Y Y)-\hat{\pi}(Y Z)=$ $\frac{4 \pi}{\left(3+\pi^{2}\right)(1+\pi)} \pi$. The RHS of both (9) and (10) become $E[Z]$. Hence, this communication strategy is indeed the equilibrium for $\lambda \geq \bar{\lambda}_{\mathrm{cl}}^{-1}$.

Now turn to gl. Assume $X_{1,1}=X_{2,1}=Y$. For given parameter values the collusion strategy is the same as the cut-off strategy in case of dl cum gl. Thus, $\hat{\pi}\left(Y Y Y Y ; \bar{y}_{\mathrm{S}}^{\mathrm{gl}}=0\right)=$ $(1+F(0)) \frac{1+\pi}{1+\pi^{2}+2 F(0) \pi} \pi=\pi(1+\pi) /\left(1+\pi^{2}\right)$, and $\hat{\pi}\left(Y Y Z Z ; \bar{y}_{\mathrm{S}}^{\mathbf{g l} *}=0\right)=0$, and the RHS of (12) becomes $E[Z]$ for $\bar{y}_{\mathrm{S}}^{\mathrm{gl} *}=0$. Hence, this collusion strategy is indeed the equilibrium strategy for $\lambda \geq \bar{\lambda}_{\mathrm{cl}}^{\mathrm{gl}}$.

Now assume $X_{1,1} \neq X_{2,1}$. Assume $N=2$, and define $a:=a_{1}$. $\hat{\pi}_{1}(Y Z Y Y ; a)=$ $\operatorname{Pr}(\bar{\theta} \mid Y Z Y Y ; a)$. Use

$$
\begin{aligned}
\operatorname{Pr}(Y Z Y Y \mid \bar{\theta} ; a) & =\frac{1}{2} \frac{1-\pi}{2}\left(\operatorname{Pr}(y>a>z \mid y>z)+\operatorname{Pr}(y>z>a \mid y>z) \frac{1}{2}+\operatorname{Pr}(a>y>z \mid y>z) \frac{1}{2}\right) \\
& =\frac{1-\pi}{4}\left(\frac{1}{2}+F(a)(1-F(a))\right) .
\end{aligned}
$$

Similarly, $\operatorname{Pr}(Y Z Y Y \mid \bar{\theta} ; a)=\frac{1}{8}-\frac{\pi}{4} F(a)(1-F(a))$. Hence, $\hat{\pi}_{1}(Y Z Y Y)=\frac{\pi}{1+\pi}\left(1+2 F(a)-2 F(a)^{2}\right)$.

Analogously, $\hat{\pi}_{1}(Y Z Z Z)=\frac{\pi}{1+\pi}\left(1-2 F(a)+2 F(a)^{2}\right)$, and the reputational gap becomes $4 \frac{\pi}{1+\pi} F(a)(1-F(a))$. As $\lambda \frac{4 \pi}{1+\pi} F(0)(1-F(0))=0<E[Z]$ and $\lambda \frac{4 \pi}{1+\pi} F(E(Z))(1-F(E[Z]))>$ 0 , for all continuous $F$ and any finite $\lambda$ there is a unique $a_{1}^{*}>0$ that satisfies (11). That is, for any finite $\lambda, N^{*} \geq 2$.

Proof of Proposition 6: Suppose $\lambda=0$. Then, $W_{\text {ia }}(0, \pi)$ is equal to $W(0, \pi)$ in case one agent reports to the center that $y \geq \bar{y}_{\mathrm{ia}}^{F B}$ or $y<\bar{y}_{\mathrm{ia}}^{F B}$. In case of $\mathrm{cl}$, two agents reveal information truthfully to the center. By continuity of $W_{\mathrm{ia}}(\lambda, \pi)$ and $W_{\mathrm{cl}}(\lambda, \pi)$ in $\lambda$, $W_{\text {ia }}(\lambda, \pi)<W_{\text {cl }}(\lambda, \pi)$ for all $\lambda<\lambda_{4}$, for some $\lambda_{4}>0$. The second part of the proposition follows from the facts that (i) $\bar{\lambda}_{\mathrm{cl}}^{\text {lo }}>\bar{\lambda}_{\text {ia }}$ (see Propositions 1 and 4), and (ii) for all $\lambda$ agents send influential information under $\mathrm{cl}$ cum gl. The truth of the final statement in the proposition has been verified numerically. 
Proof of Proposition 7: It follows from Propositions 2 and 4 that $\bar{\lambda}_{\mathrm{dl}}^{\mathrm{lo}}>\bar{\lambda}_{\mathrm{cl}}^{\mathrm{lo}}$ iff (16) holds. The existence of $\lambda_{6}$ then follows from the continuity of $W$ in $\lambda$.

Proof of Proposition 8: Consider cl, and suppose $N=3$. We know $E\left[Z \mid 0=a_{0} \leq z \leq a_{2}\right]-$ $a_{1}=E\left[Z \mid a_{1} \leq z \leq a_{3}=1\right]-a_{2}$ from (9). If two becomes the maximum number of ranks, then $a_{1}=0$, and so this equality becomes $E\left[Z \mid 0 \leq z \leq a_{2}\right]=E[Z]-a_{2}$. For any $f_{X}$, let $a_{2}^{*}<E[Z]$ denote the unique value of $a_{2}$ satisfying this equality. Let $\mathbf{a}_{2 / 3}^{*}:=\left(0,0, a_{2}^{*}, 1\right)$. Hence, (9) and (10) become $\lambda\left[\hat{\pi}\left(Y Y ; \bar{y}_{\mathrm{S}}^{\mathrm{lo}}, \mathbf{a}_{2 / 3}^{*}\right)-\hat{\pi}\left(Y Z ; \bar{y}_{\mathrm{S}}^{\mathrm{lo}}, \mathbf{a}_{2 / 3}^{*}\right)\right]=E[Z]-a_{2}^{*}$ and $E\left[Z \mid s^{Y}, s^{Y}, \bar{y}_{\mathrm{S}}^{\text {lo }}\right]-\bar{y}_{\mathrm{S}}^{\text {lo }}=E[Z]-a_{2}^{*}$. There is a unique $\bar{y}_{\mathrm{S}}^{\text {lo }}(\pi)$ that satisfies the latter equality. We can then use $\lambda\left[\hat{\pi}\left(Y Y ; \bar{y}_{\mathrm{S}}^{\text {lo }}(\pi), \mathbf{a}_{2 / 3}^{*}\right)-\hat{\pi}\left(Y Z ; \bar{y}_{\mathrm{S}}^{\text {lo }}(\pi), \mathbf{a}_{2 / 3}^{*}\right)\right]=E[Z]-a_{2}^{*}$ to find $\underline{\lambda}(\pi)$. For $\lambda \geq \underline{\lambda}(\pi)$, agents use at most two ranks. $\bar{\lambda}(\pi)$ is obtained from our numerical simulations. We checked the statement for $\pi \in[0.05,0.95]$

Proof of Proposition 9: Fix $\lambda, \pi$, and $f_{X}$. Suppose $X_{1,1}=X_{2,1}$. A straightforward comparison of (6) and (12) shows that welfare is the same under $\mathrm{dl}$ and $\mathrm{cl}$ for all $f_{X}, \pi$, and $\lambda$. Now suppose $X_{1,1} \neq X_{2,1}$. In case of $\mathrm{cl}$ and in equilibrium, the more ranks the agents use, the higher is $W$. Hence, it suffices to show that the proposition is true if communication under $\mathrm{cl}$ is limited to two ranks. Proposition 4 (iv) shows that an equilibrium with two ranks exists for all parameter values. This partition is characterized by $a_{1}^{*} \in(0, E[Z])$. Thus, if agents rank their technologies differently, the center picks the higher ranked technology. Given the communication strategies of the agents this technology is indeed the better one. However, for $(y, z) \in\left[0, a_{1}^{*}\right]^{2}$ and $(y, z) \in\left[a_{1}^{*}, 1\right]^{2}$, both technologies are ranked in the same way. Hence, the center tosses a fair coin. The inferior technology is chosen half of the time at both sites. In case of $\mathrm{dl}$, for $y<\bar{c}_{\mathrm{D}}^{*} \leq z$, the $Y$-user switches to $Z$, and the $Z$-user continues his technology. Both agents use the superior technology in $t=2$. The same holds, mutatis mutandis, for $z<\bar{c}_{\mathrm{D}}^{*} \leq y$. However, for $(y, z) \in\left[0, \bar{c}_{\mathrm{D}}^{*}\right]^{2}$, both agents switch, while if $(y, z) \in\left[\bar{c}_{\mathrm{D}}^{*}, 1\right]^{2}$, both agents continue. In either case, the inferior technology is used at one site with probability one. Clearly, if $a_{1}^{*}=\bar{c}_{\mathrm{D}}^{*}$, then $\mathrm{cl}$ and $\mathrm{dl}$ would yield the same expected welfare. For given parameter values, they are, however, not the same. $\bar{c}_{\mathrm{D}}^{*}$ satisfies $\lambda \frac{\pi}{1+\pi}=E[Z]-\bar{c}_{\mathrm{D}}^{*}($ see $(7))$, whereas $a_{1}^{*}$ satisfies $\lambda \frac{\pi}{1+\pi} 4 F\left(a_{1}^{*}\right)\left(1-F\left(a_{1}^{*}\right)\right)=E[Z]-a_{1}^{*}$ (see (14)). As $4 F\left(a_{1}\right)\left(1-F\left(a_{1}\right)\right)<1$ for all $a_{1}$, for given parameter values, the reputational gap in case of $\mathrm{cl}$ is smaller than in case of $\mathrm{dl}$. As this gap equals the size of the distortion, 
$E[Z]-a_{1}^{*}$ or $E[Z]-\bar{c}_{\mathrm{D}}^{*}$, cl yields a higher expected welfare than dl.

\section{References}

Austen-Smith, David. 1990. "Information Transmission in Debate." American Journal of Political Science, 34: 124-152.

Alonso, Ricardo, Wouter Dessein and Niko Matouschek. 2008. "When Does Coordination Require Centralization?" American Economic Review, 98 (1): 145-179.

Austen-Smith, David, and Timothy Feddersen. 2005. "Deliberation and Voting Rules." In Social Choice and Strategic Decisions: Essays in Honor of Jeffrey S. Banks, ed. David Austen-Smith and John Duggan, Berlin: Springer-Verlag.

Bala, Venkatesh, and Sanjeev Goyal. 1998. "Learning from Neighbours." Review of Economic Studies 65 (3): 595-621.

Bikhchandani, Sushil, David Hirshleifer, and Ivo Welch. 1998. "Learning from the Behavior of Others: Conformity, Fads, and Informational Cascades." Journal of Economic Perspectives, 12 (3): 151-170.

Bergemann, Dirk and Juuso Välimäki. 2006. "Bandit Problems." Cowles Foundation Discussion Paper 1551.

Çelen, Boğaçhan, Shachar Kariv, and Andrew Schotter. 2008. "An Experimental Test of Advice and Social Learning." http://emlab.berkeley.edu/ kariv/.

Coughlan, Peter J.. 2000. "In Defense of Unanimous Jury Verdicts: Mistrials, Communication, and Strategic Voting." American Political Science Review, 94 (2): 375-393.

Dessein, Wouter. 2007. "Why a Group Needs a Leader: Decision-making and Debate in Committees." http://www0.gsb.columbia.edu/faculty/wdessein/.

Eddy, David M. 1990. "Clinical Decision Making: From Theory to Practice. The Challenge." Journal of the American Medical Association. 263 (2): 287-290.

Fink, Arlene, Jacqueline Kosecoff, Mark Chassin, and Robert H. Brook 1984. "Consensus Methods: Characteristics and Guidelines for Use." American Journal of Public Health, 74 
(9): 979-983.

Friebel, Guido and Michael Raith. Forthcoming (forthcoming). "Resource Allocation and Firm Scope." American Economic Journal: Microeconomics.

Gilligan, Thomas W. and Keith Krehbiel, 1987. "Collective Decisionmaking and Standing Committees: an Informational Rationale for Restrictive Amendment Procedures", Journal of Law, Economics, and Organization, 3: 287-335.

Hayek, Friedrich A., 1945. "The Use of Knowledge in Society." American Economic Review, 35: 519-530.

Gibbons, Robert. 1992. A Primer in Game Theory. Pearson Education, Harlow, UK.

Kanodia, Chandra, Robert Bushman, and John Dickhaut. "Escalation Errors and the Sunk Cost Effect: An Explanation Based on Reputation and Information Asymmetries." Journal of Accounting Research, 27: 59-77.

Milgrom, Paul, and John Roberts. 1986. "Relying on the Information of Interested Parties." Rand Journal of Economics, 17: 18-32.

Milgrom, Paul, and John Roberts. 1994. "Comparing Equilibria." American Economic Review, 84: 441-459.

Mookherjee, Dilip. 2006. "Decentralization, Hierarchies and Incentives: A Mechanism Design Perspective." Journal of Economic Literature, 44: 367-390.

Oates, Wallece E. 1999. "An Essay on Fiscal Federalism." Journal of Economic Literature, 37: 1120-1149.

Ottaviani, Marco and Peter Norman Sørensen. 2001. "Information aggregation in debate: who should speak first?." Journal of Public Economics, 81: 393-421.

Ottaviani, Marco and Peter Norman Sørensen. 2006. "Reputational Cheap Talk." Rand Journal of Economics, 37: 155-175.

Phelps, Charles E. 2000. "Information Diffusion and Best Practice Adoption." In Handbook of Health Economics, Volume 1A, ed. Anthony J. Culyer and Joseph P. Newhouse, 223-264. Amsterdam: Elsevier, Amsterdam. 
Pochet, Phillipe. 2005. "The Open Method of Co-ordination and the Construction of Social Europe. A Historical Perspective." In The Open Method of Co-ordination in Action. The European Employment and Social Inclusion Strategies, ed. Jonathan Zeitlin and Phillipe Pochet, 37-82. Brussels: PIE-Peter Lang.

Poitevin, Michel. 2000. Can the Theory of Incentives Explain Decentralization? Canadian Journal of Economics, 33: 878-906

Prat, Andrea. 2005. "The Wrong Kind of Transparency." American Economic Review, 95: 862-877.

Prendergast, Canice and Stole. 1996. "Impetuous Youngsters and Jaded Old-Timers: Acquiring a Reputation for Learning." Journal of Political Economy, 104: 1105-1134.

Radaelli, Claudio M. 2003. "The Open Method of Coordination: a New Governance Architecture for the European Union?". SIEPS report 2003-1.

Rantakari, Heikki. 2008. "Governing Adaptation." Review of Economic Studies, 75: 12571285.

Rowe, Gene, George Wright, and Fergus Bolger. 1991. "Delphi: A Reevaluation of Research and Theory." Technological Forecasting and Social Change, 39 (3): 235-251.

Smith, Lones and Peter Sørensen. 2000. "Pathological Outcomes of Observational Learning." Econometrica, 68 (2): 371-398.

Suurmond, Guido, Otto H. Swank and Bauke Visser. 2004. "On the Bad Reputation of Reputational Concerns." Journal of Public Economics, 88: 2817-2838.

Swank, Otto H. and Bauke Visser. 2009. "Is Transparency to no Avail? Committee DecisionMaking, Pre-meetings, and Credible Deals." http://people.few.eur.nl//bvisser/.

Thaler, Richard H. 2000. "Towards a Positive Theory of Consumer Choice." Journal of Economic Behavior and Organization, 1: 39-60.

Visser, Bauke and Otto H. Swank. 2007. "On Committees of Experts." Quarterly Journal of Economics, 122 (1): 337-372.

Volden, Craig, Michael M. Ting and Daniel P. Carpenter. 2008. "A Formal Model of Learning and Policy Diffusion." American Political Science Review, 102 (3): 319-332. 